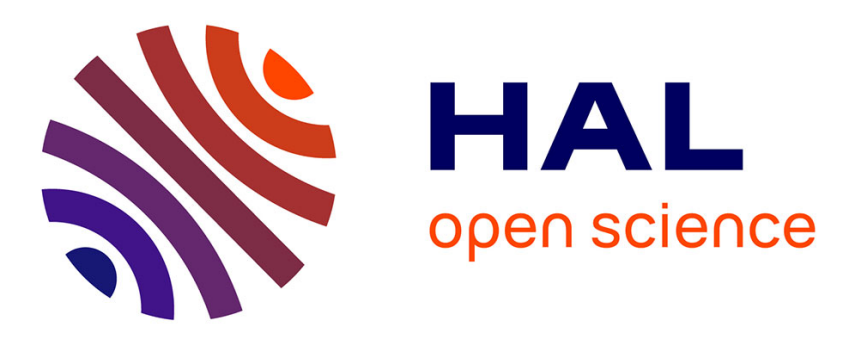

\title{
Quantum Dynamics of Electron-Hole Separation in Stacked Perylene Diimide-Based Self-Assembled Nanostructures
}

Dominik Brey, Wjatscheslaw Popp, Praveen Budakoti, Gabriele d'Avino, Irene Burghardt

\section{To cite this version:}

Dominik Brey, Wjatscheslaw Popp, Praveen Budakoti, Gabriele d'Avino, Irene Burghardt. Quantum Dynamics of Electron-Hole Separation in Stacked Perylene Diimide-Based Self-Assembled Nanostructures. Journal of Physical Chemistry C, 2021, 125 (45), pp.25030 - 25043. 10.1021/acs.jpcc.1c06374 . hal-03441981

\section{HAL Id: hal-03441981 \\ https://hal.science/hal-03441981}

Submitted on 22 Nov 2021

HAL is a multi-disciplinary open access archive for the deposit and dissemination of scientific research documents, whether they are published or not. The documents may come from teaching and research institutions in France or abroad, or from public or private research centers.
L'archive ouverte pluridisciplinaire HAL, est destinée au dépôt et à la diffusion de documents scientifiques de niveau recherche, publiés ou non, émanant des établissements d'enseignement et de recherche français ou étrangers, des laboratoires publics ou privés. 


\title{
Quantum Dynamics of Electron-Hole Separation in Stacked Perylene Diimide-Based Self-Assembled Nanostructures
}

\author{
Published as part of The Journal of Physical Chemistry virtual special issue "125 Years of The Journal of \\ Physical Chemistry".
}

\author{
Dominik Brey, Wjatscheslaw Popp, Praveen Budakoti, Gabriele D’Avino, and Irene Burghardt*
}

Cite This: https://doi.org/10.1021/acs.jpcc.1c06374

ABSTRACT: We report on high-dimensional quantum dynamical simulations of electron-hole separation in self-assembled mesomorphic nanostructures composed of donor-acceptor conjugated co-oligomers. The latter are based on perylene diimide (PDI) acceptor units combined with fluorene-thiophene-benzothiadiazole donor units, which form highly ordered, stacked structural motifs upon self-assembly. Simulations are shown for a first-principles parametrized model lattice of 25 stacked PDI units under the effects of an applied external field and temperature. The simulations are carried out with the multilayer multiconfiguration timedependent Hartree (ML-MCTDH) method with nearly 900 vibrational degrees of freedom and 25 electronic states. Temperature effects are included using the thermofield dynamics approach. A transition between a

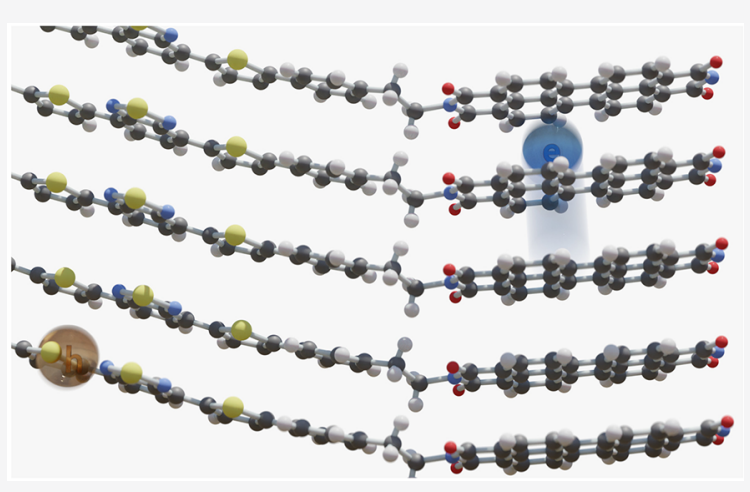
short-time coherent dynamics and a kinetic regime is highlighted. From a flux-over-population analysis, electron-hole dissociation rates are obtained in the range of 5-20 ns ${ }^{-1}$ in the absence of static disorder, exhibiting a moderate field and temperature dependence. These results for electron-hole separation rates can be employed as a benchmark to calibrate the parametrization of kinetic Monte Carlo simulations applied to much larger lattice sizes.

\section{INTRODUCTION}

Photoinduced charge separation in organic donor-acceptor (DA) systems is often treated by Onsager-Braun theory ${ }^{1,2}$ or improved variants of this theory ${ }^{3-6}$ and by kinetic Monte Carlo (KMC) modeling. ${ }^{7-9}$ These approaches capture the main features of thermally induced breakup of interfacial electronhole $(\mathrm{e}-\mathrm{h})$ pairs under the effect of the $\mathrm{e}-\mathrm{h}$ Coulomb interaction that is modified by the dielectric screening effects of the local environment. However, the specificities of the interface morphology, along with quantum effects may play a non-negligible role in the charge separation process and can modify the relevant time scales of the elementary events. Indeed, the dissociation of bound interfacial $\mathrm{e}-\mathrm{h}$ pairs seems far more efficient in many systems than predicted by Onsager-Braun theory. $6,10,11$

These deviations from the standard theory are most conspicuous in the context of ultrafast charge separation that has been observed, e.g., in regioregular fullerene-based organic donor-acceptor systems. ${ }^{12,13}$ Here, the delocalization of charge carriers in the fullerene domain may play a prominent role, ${ }^{11,14}$ promoting ultrafast long-range charge separation. Similar observations have been made for phthalocyanine acceptor species, ${ }^{15,16}$ and charge delocalization has also been shown to occur in perylene diimide (PDI) acceptors. ${ }^{17-19}$ Such delocalization effects could also affect slower charge separation processes, by modifying the localization length of the charge separated states and hence the energetics of the process. ${ }^{15,20}$ To some extent, such effects can be included in the abovementioned rate theories. ${ }^{6,10,11}$ Finally, vibronically hot e-h pairs could play a role in ultrafast charge separation steps. However, in most relevant systems, excess energy does not seem to have a predominant effect, and relaxed interfacial $\mathrm{e}-\mathrm{h}$ species emerge from the initial exciton dissociation. ${ }^{11,21}$

In the present study, we carry out a full quantum dynamical analysis $^{22}$ of charge separation in mesomorphic self-assembled nanostructures composed of conjugated donor-acceptor block co-oligomers. Conjugated block copolymer photovoltaics have emerged as a route toward highly controlled heterojunction architectures. $^{23-25}$ Specifically, the mesomorphic DA block cooligomer nanostructures under consideration in the present work are based on PDIs combined with fluorene-thiophene-

Received: July 16, 2021

Revised: October 2, 2021 
(a)

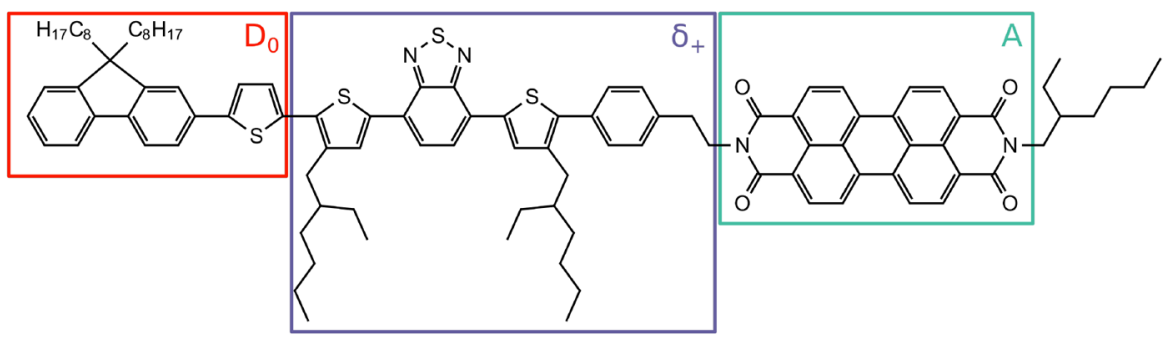

(b)

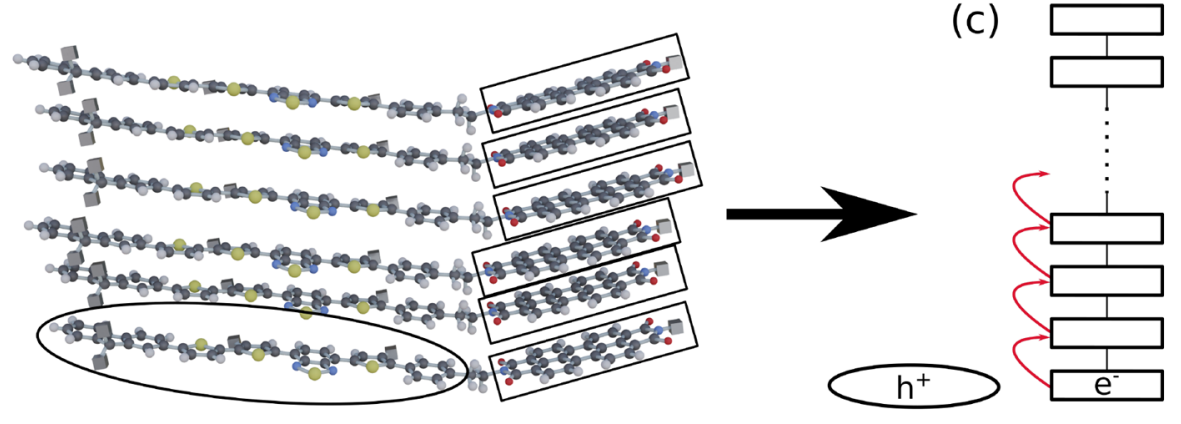

Figure 1. (a) Donor-acceptor dyad denoted as $\mathrm{D}_{0} \delta_{+} \mathrm{A}$ (see nomenclature of ref 31 ). The $\delta_{+}$spacer containing a benzothiadiazol moiety is sandwiched between the fluorene-thiophene donor and the PDI acceptor. (b) Self-assembled mesomorphic donor-acceptor co-oligomer nanostructure described in ref 29. (c) Model employed in the present simulations, where charge separation is investigated for a finite 1D lattice of stacked PDI units Coulombically coupled to a single donor site where a hole is assumed to be stationary. This model is suitable to investigate electron migration under the influence of the $\mathrm{e}-\mathrm{h}$ interaction in the present system where hole migration is 3 orders of magnitude slower than electron migration. ${ }^{32}$

benzothiadiazole donor units. Such self-assembled nanostructures have been described in refs $26-29$ and were characterized by electron diffraction (ED) studies. ${ }^{29}$ Here, we focus on a particular, zipper-like structural motif characterized in ref 29 and employ an effective $\mathrm{e}-\mathrm{h}$ potential that is based upon a microelectrostatic (ME) analysis ${ }^{30}$ to quantify the effective e$\mathrm{h}$ barrier to charge separation. For the present DA system, this permits a precise characterization of the energetics of the charge separation process. Furthermore, the relevant DA dyad units were previously characterized in solution phase in a combined spectroscopic and theoretical study, ${ }^{31}$ yielding insight into the photochemical steps preceding charge separation.

Since it turns out that hole migration is far slower than electron migration in the system under study, ${ }^{32}$ we focus on the electron migration process and describe effective one-dimensional (1D) transport along the PDI stacking direction. Given that the $\mathrm{e}-\mathrm{h}$ separation is comparatively slow, a rate computation by the flux-over-population method $^{33-35}$ is employed in conjunction with a quantum dynamical approach. The computed rates in the absence of static disorder fall into the range of $5-20 \mathrm{~ns}^{-1}$. These results for $\mathrm{e}-\mathrm{h}$ separation rates are employed as a benchmark to calibrate the parametrization of KMC simulations applied to much larger lattice sizes, to be reported in a follow-up paper.

The quantum dynamical treatment of $\mathrm{e}-\mathrm{h}$ separation is highly challenging since electron-phonon (vibronic) coupling plays an important role, requiring a nonperturbative treatment. In addition, the inclusion of temperature effects is demanding, which is here realized using the thermofield dynamics (TFD) approach. ${ }^{3-41}$ In the present study, we employ highly efficient multiconfigurational methods, notably the multilayer multiconfiguration time-dependent Hartree (ML-MCTDH) meth$\mathrm{od}^{42-46}$ to treat nearly 900 vibrational degrees of freedom and 25 electronic states, within a linear vibronic coupling (LVC) model. $^{22,47}$
In the following, we first describe the donor-acceptor architecture under study (section 2), followed by a description of the vibronic lattice Hamiltonian based on electronic structure information and ME calculations, a summary of the quantum dynamical methodology, and our approach to rate computation (section 3). Following this, we turn to the results (section 4), that represent a first-principles computation of $\mathrm{e}-\mathrm{h}$ separation rates as a function of temperature and field strengths. Section 5 concludes with a discussion and perspectives.

\section{DONOR-ACCEPTOR CO-OLIGOMER ARCHITECTURE}

The DA block co-oligomer system under study, illustrated in Figure 1, belongs to a class of covalently bound DA dyad assemblies combining PDI acceptor moieties with various types of donor units. ${ }^{25-29,48,49}$ Architectures that rely on the selfassembly of such co-oligomers are promising since they should permit the controlled formation of $\mathrm{e}-\mathrm{h}$ pairs followed by efficient carrier transport along 1D or two-dimensional (2D) conductive channels. In practice, though, these approaches have mostly been limited to power conversion efficiencies (PCE) of $1-2 \%$, inferior to conventional bulk heterojunction architectures. However, a PCE of $2.7 \%$ was achieved ${ }^{27}$ by suitably tuning the band gap of the donor and optimizing the molecular packing. Since then, various dyad and triad species $\left(\mathrm{DA},{ }^{28} \mathrm{DAD},{ }^{50,81}\right.$ $\mathrm{ADA}^{28,48}$ ) have been explored, which were prepared in liquid crystalline phases or lamellar nanostructures. ${ }^{28,48,50,51}$ More recently, various types of junction-functionalized block cooligomers with related constituents were investigated. ${ }^{52}$ The latter study highlights geminate recombination as the dominant loss channel.

In an effort to explore and optimize the chemical design of the DA building blocks, various DA dyads were synthesized and investigated in combined spectroscopic and computational studies. ${ }^{31}$ Specifically, the donor species, generally based upon thiophene and fluorene units, was modified such as to add 
electron-donating or -withdrawing groups which permit tuning of the donor properties. The key aim was to achieve long-lived charge transfer (CT) states and reduce recombination, which could be accomplished to some extent, such that CT state lifetimes of several nanoseconds were obtained. ${ }^{31}$ The CT formation times were typically of the order of $10-100 \mathrm{ps}$, compatible with small electronic couplings of the order of 1-2 $\mathrm{meV}$. However, these studies were carried out in solution phase, such that the validity of these conclusions for varying types of molecular packing remained open. Indeed, preliminary timeresolved spectroscopic investigations of the thin-film material provided evidence for unforeseen photoproducts and a kinetics that was not compatible with the solution phase. ${ }^{32}$

The system at the center of the present study, depicted in Figure 1a) and denoted as $\mathrm{D}_{0} \delta_{+} \mathrm{A}$ following the nomenclature of ref 31 , combines a fluorene-thiophene donor part $\left(D_{0}\right)$ and a PDI acceptor part $(\mathrm{A})$ with a benzothiadiazol containing $\left(\delta_{+}\right)$ spacer moiety. The latter has been shown to significantly affect the photochemistry of the system. In the absence of the $\delta_{+}$ moiety, $\mathrm{e}-\mathrm{h}$ formation proceeds in a two-step process, where excitation energy transfer from the donor to the acceptor occurs on a subpicosecond scale, followed by slower hole transfer from the PDI acceptor to the donor within less than $10 \mathrm{ps}^{31}$ In contrast, the presence of the $\delta_{+}$moiety leads to direct DA charge transfer on a slower time scale, around $0.1 \mathrm{~ns}$. More precisely, the time scale for charge formation and geminate recombination were determined as $\tau_{\text {form }}=90$ ps and $\tau_{\text {rec }}=410$ ps from timeresolved spectroscopy of the $\mathrm{D}_{0} \delta_{+} \mathrm{A}$ species in chloroform. ${ }^{31}$ In view of the slow formation of the CT state, the present study does not explicitly address the exciton dissociation step, but sets out to characterize the $\mathrm{e}-\mathrm{h}$ pair separation starting from the initially formed CT state of the DA dyad (i.e., $\left(\mathrm{D}_{0} \delta_{+}\right)^{+} \mathrm{A}^{-}$).

For the $\mathrm{D}_{0} \delta_{+} \mathrm{A}$ based material, mobility measurements were carried out using an organic field-effect transistor (OFET) setup, yielding a moderate electron mobility $\left(\mu_{\mathrm{e}}=0.02 \mathrm{~cm}^{2} /(\mathrm{V} \mathrm{s})\right)$ and a much smaller hole mobility $\left(\mu_{\mathrm{h}}=3.3 \times 10^{-5} \mathrm{~cm}^{2} /(\mathrm{V} \mathrm{s})\right) .^{32}$ Ambipolar transport with balanced electron vs hole mobilities was achieved for longer donor moieties. ${ }^{32}$ As a result, we focus here entirely on the electron transport in $\mathrm{D}_{0} \delta_{+} \mathrm{A}$ based assemblies and assume that the hole rests immobile.

\section{METHODS}

In the following, we first introduce a vibronic lattice Hamiltonian suitable to describe the $\mathrm{e}-\mathrm{h}$ separation process (section 3.1), along with its expanded version employed in the context of the thermofield dynamics approach (section 3.2). Next, the quantum dynamical setup is described (section 3.3), and the rate computation approach is summarized (section 3.4).

3.1. Vibronic Hamiltonian for $\mathrm{e}-\mathrm{h}$ Separation. In the present work, an electronic-vibrational wave function description is employed in order to obtain time-dependent site populations for a finite-dimensional $\mathrm{e}-\mathrm{h}$ lattice whose spatial extension matches the effective Coulomb barrier to charge separation. In practice, a minimal lattice size is chosen (i.e., $N=$ $25 \mathrm{e}-\mathrm{h}$ states involving 25 acceptor (PDI) sites coupled to a single donor site), as illustrated in Figure 1c). As mentioned above, hole migration is not included in the present treatment since hole mobility is known to be smaller by 3 orders of magnitude as compared with electron mobility. ${ }^{32}$ Due to the presence of an external field, the process is effectively unidirectional such that the model of Figure 1c) is adequate.
The Hamiltonian $H$ can be separated into an electronic part $H^{\mathrm{el}}$, the interaction $H^{\text {field }}$ with an external field, and a phonon part $H^{\mathrm{ph}}$ along with the electron-phonon coupling $H^{\mathrm{el}-\mathrm{ph}}$,

$$
H=H^{\mathrm{el}}+H^{\text {field }}+H^{\mathrm{ph}}+H^{\mathrm{el}-\mathrm{ph}}
$$

The Hamiltonian is set up in a basis of e-h states $\left|\nu_{\mathrm{e}}, \eta_{\mathrm{h}}\right\rangle$ representing an electron located at site $\nu_{\mathrm{e}}$ and a hole located at site $\eta_{\mathrm{h}}$. In the present system, the hole location is assumed to be fixed at $\eta_{\mathrm{h}}=0_{\mathrm{h}}$ (assuming negligible hole mobility on the time scale of observation), while the electron location is variable at $\nu_{\mathrm{e}}$ $=n$. Therefore, we specify a series of charge-separated (CS) states as $|n\rangle=\left|\nu_{\mathrm{e}}=n, \eta_{\mathrm{h}}=0_{\mathrm{h}}\right\rangle$. In the following, these states are also referred to as $\operatorname{CS}(n)$ states. Here, the $\operatorname{CS}(0)$ state refers to the initial state where the electron and hole are located on a covalently bound DA dyad; this is the bound "interfacial" $\mathrm{e}-\mathrm{h}$ state denoted CT in the above discussion. The $\mathrm{e}-\mathrm{h}$ basis as specified here represents a diabatic basis ${ }^{22}$ in the sense that it is property-based and that potential-type couplings appear.

Hence, the electronic Hamiltonian is comprised of $N=25$ diabatic states which are coupled via the electron transfer integral $t_{\mathrm{e}}$

$$
H^{\mathrm{el}}=\sum_{n=0}^{N-1} \beta_{n}|n\rangle\langle n|+\sum_{n=0}^{N-2} t_{\mathrm{e}}(|n\rangle\langle n+1|+| n+1\rangle\langle n|)
$$

The on-site energies $\beta_{n}$ were computed by ME calculations ${ }^{30}$ describing the separated $\mathrm{e}-\mathrm{h}$ pair in the local electrostatic environment (see Supporting Information section S1) for the zipper-like structural motif mentioned above. ${ }^{29}$ For the purpose of the present quantum dynamical analysis, a 1D simplified fit of the ME binding energy is employed along the PDI stacking direction, with the Coulombic functional form $V(r)=E_{0}-e^{2} /\left(4 \pi \epsilon_{0} \epsilon_{r}^{\text {eff }}\left(r-r_{0}\right)\right)$ where the reference energy is given as $E_{0}=0.0167 \mathrm{eV}$, the effective relative permittivity factor is $\epsilon_{r}=5.53$, and the reference distance $r_{0}=14.22 \AA$ refers to the minimal $\mathrm{e}-\mathrm{h}$ distance defined by a stacked DA fragment. The resulting on-site energies are listed in Table 1 (see

Table 1. Values of the On-Site Energies $\beta_{n}(\mathrm{eV})$

\begin{tabular}{l|rrrrr}
$n$ & 0 & 1 & 2 & 3 & 4 \\
$\beta_{n}$ & -0.4338 & -0.4338 & -0.3419 & -0.2965 & -0.2518 \\
\hline$n$ & 5 & 6 & 7 & 8 & 9 \\
$\beta_{n}$ & -0.2052 & -0.1701 & -0.1402 & -0.1181 & -0.0999 \\
\hline$n$ & 10 & 11 & 12 & 13 & 14 \\
$\beta_{n}$ & -0.0860 & -0.0744 & -0.0652 & -0.0573 & -0.0509 \\
\hline$n$ & 15 & 16 & 17 & 18 & 19 \\
$\beta_{n}$ & -0.0453 & -0.0407 & -0.0365 & -0.0330 & -0.0298 \\
\hline$n$ & 20 & 21 & 22 & 23 & 24 \\
$\beta_{n}$ & -0.0271 & -0.0246 & -0.0224 & -0.0205 & -0.0187
\end{tabular}

Supporting Information section $\mathrm{S} 1$ for the corresponding $\mathrm{e}-\mathrm{h}$ distances). The transfer integral, $t_{\mathrm{e}}=0.097 \mathrm{eV}$, was computed by a diabatization procedure ${ }^{53}$ in conjunction with supermolecular TDDFT calculations for a dimer composed of two stacked DA dyads, using the $\omega \mathrm{B} 97 \mathrm{XD}$ functional with a def2-SVP basis set, within the Gaussian09D package (see Supporting Information 

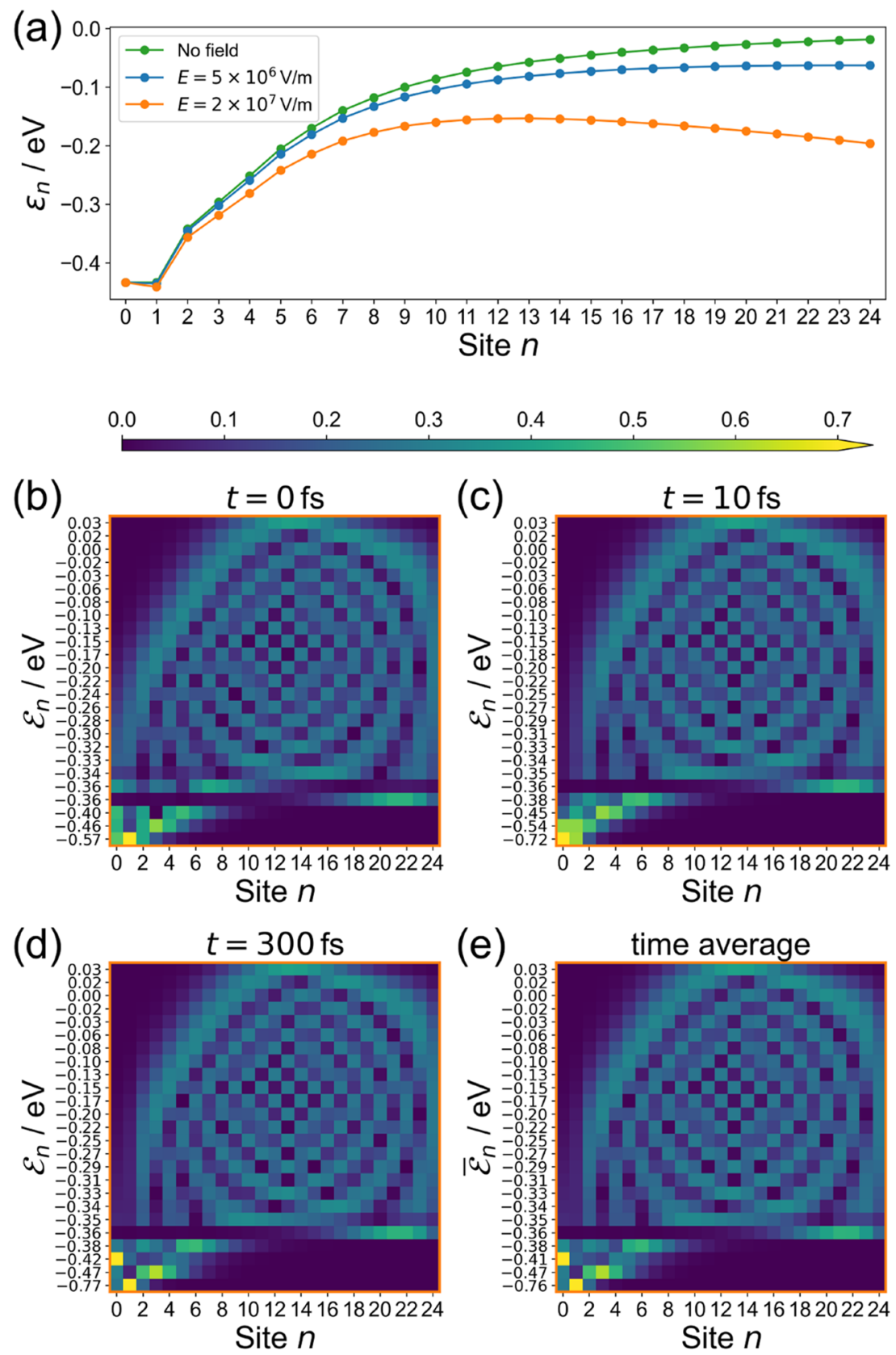

Figure 2. (a) Effective Coulomb barriers $\left\{\varepsilon_{n}\right\}$ in the absence of a field and for the two most relevant field strengths; these are constructed from a fit to a microelectrostatic analysis reported in the Supporting Information (section S1). Due to the specific spatial arrangement shown in Figure 1, the two lowest-energy charge-separated states, i.e., the intra-dyad state $\operatorname{CS}(0)$ and the nearest-neighbor state CS(1), are nearly degenerate (see Supporting Information section $S 1)$. (b-e) Vibrationally averaged electronic eigenvectors in a site-based representation (absolute values of the eigenvector coefficients) pertaining to the Hamiltonian in eq 21 , at $T=300 \mathrm{~K}$, shown for $E=2 \times 10^{7} \mathrm{Vm}^{-1}$ at different times: (b) $t=0 \mathrm{fs},(\mathrm{c}) t=10 \mathrm{fs}$, (d) $t=300 \mathrm{fs}$, and $(\mathrm{e})$ as a time average. Averaged eigenvalues $\mathcal{E}_{n}$ are indicated in ascending order along the ordinate.

section S2). Transfer integrals in PDI assemblies are known to be highly sensitive to the molecular stacking pattern., 94

The interaction with the external field applied in the PDI stacking direction is given as

$$
H^{\text {field }}=-\sum_{n=0}^{N-1} n e \Delta r E|n\rangle\langle n|
$$

where $e$ is the electronic unit charge and $\Delta r=3.72 \AA$ is the PDI stacking distance. Typical field strengths in the range of $E=10^{6}-$ $10^{7} \mathrm{~V} / \mathrm{m}$ are considered.
When combining eqs 2 and 3 , the effective on-site energies $\varepsilon_{n}$ appear:

$$
\varepsilon_{n}=\beta_{n}-n e \Delta r E
$$

which are depicted in Figure 2a) for two selected values of the electric field (i.e., $E=5 \times 10^{6}$ and $2 \times 10^{7} \mathrm{Vm}^{-1}$, where the former value corresponds to a typical experimental setup). ${ }^{28,29,32}$ Figure $2 b-e$ shows effective electronic eigenvectors that will be discussed in section 4.1. The $\varepsilon_{n}$ values define an effective Coulomb barrier to charge separation. 


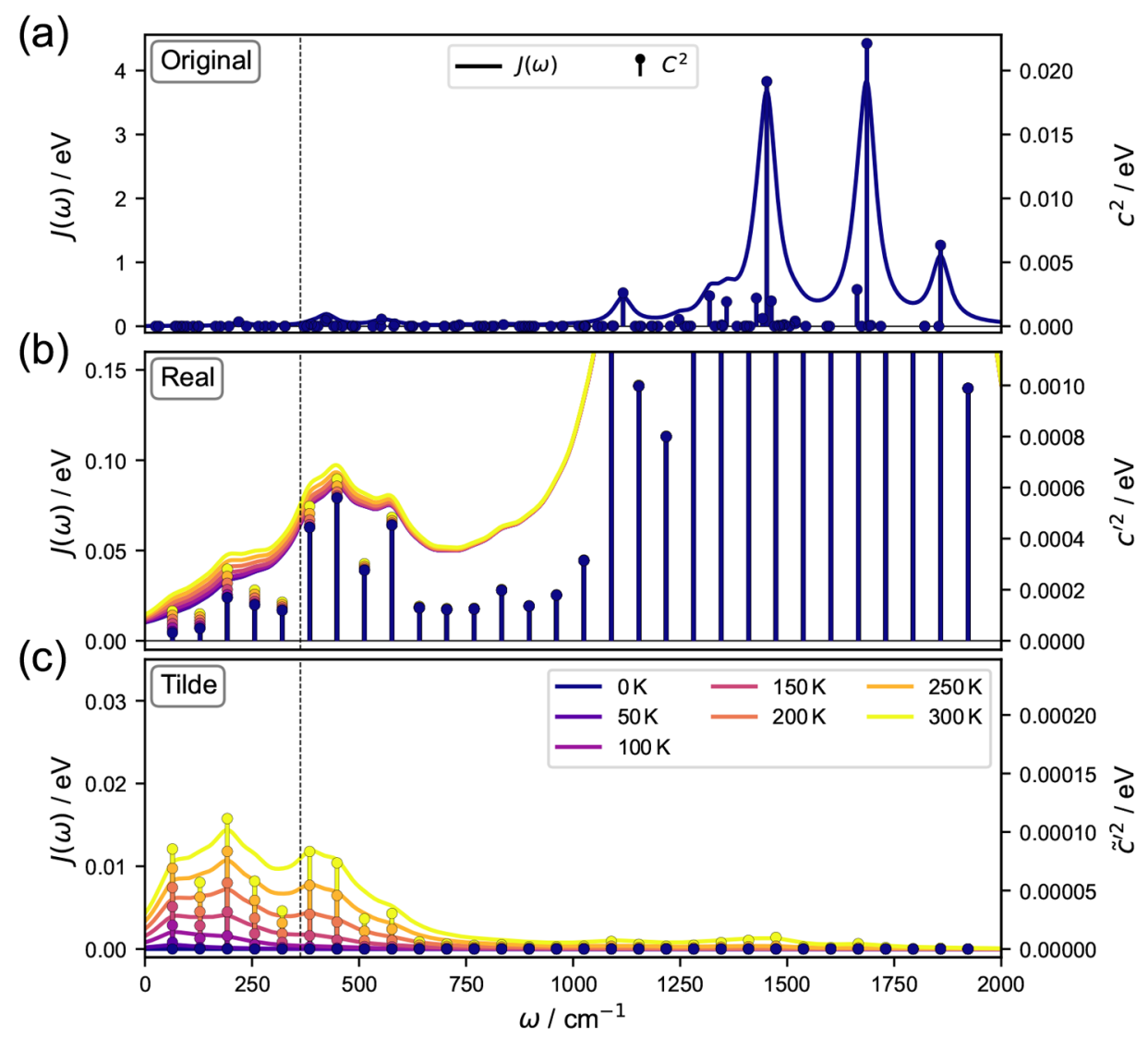

Figure 3. Spectral density of the acceptor (PDI) moiety: (a) SD based upon the original set of computed vibronic couplings $\left\{c_{n, j}^{\mathrm{A}}\right\}$ in the full normalmode space, where the envelope function corresponds to the continuous spectral density functions resulting from convolution with a Lorentzian according to eq 8. (b) Equidistant rediscretization of eq 8 for the physical part of the SD at finite temperature, with TFD couplings $c_{i}^{\prime}$ according to eq 13. (c) Likewise, rediscretization for the tilde part of the SD, with couplings $\tilde{c}_{i}^{\prime}$. A series of temperatures are shown for the TFD couplings. Note that panels (b) and (c) focus on the low-frequency part of the SD. The rediscretized real SD closely follows the original SD, except for the temperature-dependent low-frequency tail shown explicitly in panel (b); by contrast, the tilde part of the SD takes vanishing values beyond the low-frequency part. The dashed vertical line indicates the frequency cutoff below which a TFD treatment is carried out in the quantum dynamical calculations, i.e., the five lowestfrequency modes of the rediscretized representation.

Finally, the vibronic part of the Hamiltonian is treated within an LVC model ${ }^{22,47}$ which accounts for the different equilibrium geometries of neutral and charged states within a shifted harmonic-oscillator approximation for the intramolecular modes. The LVC Hamiltonian reads for $N M_{\mathrm{A}}$ acceptor modes

$$
\begin{aligned}
& H_{\mathrm{A}}^{\mathrm{ph}}+H_{\mathrm{A}}^{\mathrm{el}-\mathrm{ph}}= \\
& \quad \sum_{n=0}^{N-1} \sum_{j=1}^{M_{\mathrm{A}}}\left[\frac{1}{2} \omega_{n, j}^{\mathrm{A}}\left(\left(p_{n, j}^{\mathrm{A}}\right)^{2}+\left(q_{n, j}^{\mathrm{A}}\right)^{2}\right)+c_{n, j}^{\mathrm{A}} q_{n, j}^{\mathrm{A}}|n\rangle\langle n|\right]
\end{aligned}
$$

and for $M_{D}$ donor modes

$$
\begin{aligned}
H_{\mathrm{D}}^{\mathrm{ph}} & +H_{\mathrm{D}}^{\mathrm{el}-\mathrm{ph}}=\sum_{j=1}^{M_{\mathrm{D}}}\left[\frac{1}{2} \omega_{j}^{\mathrm{D}}\left(\left(p_{j}^{\mathrm{D}}\right)^{2}+\left(q_{j}^{\mathrm{D}}\right)^{2}\right)+\sum_{n=0}^{N-1} c_{j}^{\mathrm{D}} q_{j}^{\mathrm{D}}|n\rangle\langle n|\right] \\
= & \sum_{j=1}^{M_{\mathrm{D}}}\left[\frac{1}{2} \omega_{j}^{\mathrm{D}}\left(\left(p_{j}^{\mathrm{D}}\right)^{2}+\left(q_{j}^{\mathrm{D}}\right)^{2}\right)+c_{j}^{\mathrm{D}} q_{j}^{\mathrm{D}}\right]
\end{aligned}
$$

where mass and frequency weighted coordinates are employed and $\left\{c_{n, j}^{\mathrm{A}}\right\}$ and $\left\{c_{j}^{\mathrm{D}}\right\}$ denote vibronic couplings pertaining to the acceptor versus donor fragments. As emphasized in the last line of eq 6, the donor part of the Hamiltonian turns out to be separable because the vibronic interactions $\left\{c_{j}^{\mathrm{D}}\right\}$ act identically on all e-h states $|n\rangle, \sum_{n} c_{j}^{\mathrm{D}} q_{j}^{\mathrm{D}}|n\rangle\langle n|=c_{j}^{\mathrm{D}} q_{j}^{\mathrm{D}}$. This implies that the electronic transport is exclusively mediated by fluctuations of the acceptor vibrations while the hole carrying donor moiety can be considered static. As a result, the donor modes can be omitted from the quantum dynamical treatment, and we use the following for the vibronic part of eq 1 :

$$
H^{\mathrm{ph}}+H^{\mathrm{el}-\mathrm{ph}}=H_{\mathrm{A}}^{\mathrm{ph}}+H_{\mathrm{A}}^{\mathrm{el}-\mathrm{ph}}
$$

In a more general setup where hole transport and the photogenerated donor exciton are included, ${ }^{22}$ the interplay of donor and acceptor vibronic interactions will give rise to a more complex picture, necessitating to include the donor modes.

Even in the absence of the donor modes (i.e., $M_{D}=234$ modes in the present case), the total number of intramolecular modes of the acceptor molecules amounts to $N M_{\mathrm{A}}=25 \times 132=3300$ modes for a 25 site system, which exceeds the capacity of the present simulation setup. Therefore, a reduction strategy has to be employed, in order to reduce the number of modes by a factor of 4-5. Several such procedures exist, e.g., the selection of the most relevant modes, ${ }^{4,55}$ the construction of effective modes, ${ }^{22,56,57}$ and the sampling from a continuous spectral density (SD), ${ }^{58-60}$ which could be either of simple analytical form or else obtained from a molecular-level analysis. ${ }^{59,60}$ In the 
present work, we adopt the latter procedure, which has been previously employed to construct multistate vibronic coupling models using structured SDs. Here, a continuous SD is generated from the original set of normal modes and subsequently resampled for a reduced set of equidistant modes. $^{59}$

In further detail, vibronic couplings $\left\{c_{j}^{\mathrm{A}}\right\}$ (and $\left\{c_{j}^{\mathrm{D}}\right\}$ ) are initially computed for the full set of normal modes of the acceptor (and donor) species. These vibronic couplings were obtained from electronic structure calculations at the DFT/ $\omega$ B97XD level with def2-SVP basis set, by choosing the equilibrium geometry of the neutral species as reference and computing the gradient of the potential energy surface (PES) of the charged species at this geometry. The PES gradients are projected onto the normal modes of the neutral species, yielding directly the vibronic couplings within an LVC model. ${ }^{22}$ The reorganization energies derived from the vibronic couplings (i.e., $\left.\lambda=\sum_{j} c_{j}^{2} /\left(2 \hbar \omega_{j}\right)\right)$ are given as $\lambda_{\mathrm{A}}=0.194 \mathrm{eV}$ for the acceptor and $\lambda_{\mathrm{D}}=0.256 \mathrm{eV}$ for the donor species. A complementary procedure where the equilibrium geometry of the charged species is taken as reference and the gradients are computed with respect to the PES of the neutral species, yields similar results (see Supporting Information section S3).

From the initial set of vibronic couplings in the full normalmode basis, a state-specific, discretized SD is obtained ${ }^{22}$ which is subsequently convoluted with a Lorentzian broadening function, here for the acceptor species:

$$
\begin{aligned}
J_{n, \mathrm{~A}}(\omega) & =\frac{\pi}{2} \sum_{j=1}^{M_{A}}\left(c_{n, j}^{\mathrm{A}}\right)^{2} \delta\left(\omega-\omega_{n, j}^{\mathrm{A}}\right) \\
& \simeq \frac{\pi}{2} \sum_{j=1}^{M_{\mathrm{A}}} \frac{\left(c_{n, j}^{\mathrm{A}}\right)^{2}}{\pi} \frac{\Delta}{\left(\omega-\omega_{n, j}^{\mathrm{A}}\right)^{2}+\Delta^{2}}
\end{aligned}
$$

where the parameter $\Delta \approx 25 \mathrm{~cm}^{-1}$ determines the Lorentzian width which was chosen as about twice the root-mean-square (RMS) of the frequency spacings of the original data set ${ }^{59}$ (see Supporting Information section S3 for details). The resulting continuous SD is shown in Figure 3a). The broadening accounts for environmental effects in a condensed-phase environment, including homogeneous and heterogeneous broadening effects due to intermolecular interactions, conformational flexibility due to substituents, and modes that are not explicitly included (e.g., interfragment modes). (The same effects lead to strongly broadened absorption and emission spectra in the thin film material $^{32,49}$ where at most dominant high-frequency progressions remain visible.) Finally, the broadened spectral density is rediscretized to yield the desired number of modes with an equidistant spacing in the frequency domain, which were employed in the Hamiltonian of eq 7. To this end, equidistant frequencies $\omega_{n, j}^{\prime}=k \Delta \omega$ are introduced, whose vibronic couplings are defined in terms of the SD of eq 8 , $c_{n, j}^{\mathrm{A}^{\prime}}=\left((2 / \pi) J\left(\omega_{n, j}^{\prime}\right) \Delta \omega\right)^{1 / 2} \cdot 58,59$ Using $M_{\mathrm{A}}^{\prime}=30$ sampling points, the full number of modes entering the dynamical calculations is reduced to $N M_{A}^{\prime}=750$. Since the equidistant sampling results in a Poincaré recurrence effect, ${ }^{58,59,61}$ with a time scale of $\tau_{\mathrm{P}}=2 \pi / \Delta \omega=517$ fs (for $\Delta \omega=0.008 \mathrm{eV}$ ), we further introduced a randomization of the sampled frequencies within a narrow interval around the set of equidistant frequencies, as detailed in Supporting Information section S3.
The rediscretized SD conserves the above-mentioned reorganization energies to a good approximation ( $>90 \%)$.

3.2. Thermofield Dynamics. To include thermal effects in our model, we employ the thermofield dynamics (TFD) approach $^{36-41}$ to describe the time evolution from an initial vibronic state including thermal excitation of the low-frequency vibrational modes. In this approach, thermal ensemble averages are represented in terms of a single, temperature-dependent quantum state $\left|\psi_{T}\right\rangle$ in an extended, "doubled" Hilbert space:

$$
\left|\psi_{T}\right\rangle=Z^{-1 / 2}(T) \sum_{j} \exp \left(-\frac{E_{j}}{k_{\mathrm{B}} T}\right)\left|\varphi_{j}\right\rangle \otimes\left|\tilde{\varphi}_{j}\right\rangle
$$

where $Z$ is the partition function and $\left|\varphi_{j}\right\rangle \otimes\left|\tilde{\varphi}_{j}\right\rangle \equiv\left|\varphi_{j}, \tilde{\varphi}_{j}\right\rangle$ represents a direct product of eigenstates $\left|\varphi_{j}\right\rangle$ of the Hamiltonian, $H\left|\varphi_{j}\right\rangle=E_{j}\left|\varphi_{j}\right\rangle$, and auxiliary ("tilde") states $\left|\tilde{\varphi}_{j}\right\rangle$ obeying $\tilde{H}\left|\tilde{\varphi}_{j}\right\rangle=E_{j}\left|\tilde{\varphi}_{j}\right\rangle$. Here, $\tilde{H}$ (the "tildian") is constructed from transformation rules for bosonic or Fermionic operators. ${ }^{37,38,41}$ In this representation, the time evolution is defined in terms of the thermal Hamiltonian $H_{T}=H-\tilde{H}$ (i.e., $\left.i \hbar \partial \psi / \partial t=H_{T} \psi\right)$.

Over recent years, the TFD approach has found increased interest in the molecular physics community. ${ }^{39-41,62-64}$ In the context of thermal vibrational states, it was shown that the equations of motion can be reformulated using a thermal Bogoliubov transformation, ${ }^{38-40,65}$ permitting to use zerotemperature initial conditions instead of the thermal initial conditions of eq 9 , that is

$$
i \hbar \frac{\partial}{\partial t}|\psi\rangle=H_{T}^{\theta}|\psi\rangle \quad|\psi(t=0)\rangle=\left|\varphi_{0}\right\rangle \otimes\left|\tilde{\varphi}_{0}\right\rangle
$$

where the Bogoliubov transformed TFD Hamiltonian is given as $^{38,41}$

$$
H_{T}^{\theta}=\mathrm{e}^{i G(\theta)} H_{T} \mathrm{e}^{-i G(\theta)}
$$

For a single vibrational mode (i.e., boson degree of freedom), the Bogoliubov transformation reads as follows (complementary to the more frequent formulation in terms of creation and annihilation operators): ${ }^{38,39,41}$

$$
\exp (i G(\theta))=\exp (i \theta(q \tilde{p}+p \tilde{q})) \quad \theta=\operatorname{arctanh}\left(\mathrm{e}^{-\omega /\left(2 k_{\mathrm{B}} T\right)}\right)
$$

illustrating that the transformation mixes the coordinate $(q)$ and momentum $(p)$ variables in the original and tilde spaces; here, mass- and frequency-weighted coordinates were used as above. This is the mechanism that induces thermalization in the TFD picture.

In the present context, we adopt eq 10 as the formulation of the time-dependent Schrödinger equation, following refs 39-41. With 750 physical vibrational modes, this approach would lead to a considerable numerical effort. To mitigate the effort without significantly compromising the accuracy, the TFD approach is only applied to low frequency vibrational modes that are expected to show a significant thermal population at room temperature. In this low-frequency space, we include all modes with frequencies up to $45 \mathrm{meV}$ corresponding to $1.75 k_{\mathrm{B}} T$ at room temperature, leading to five PDI modes that are thermalized. This low frequency space is doubled into a real part and a dual part, whose vibronic couplings $c_{n, i}^{\prime}$ and $\tilde{c}_{n, i}^{\prime}$ are constructed from the original couplings $c_{n, i}$ as ${ }^{39,40}$ 


$$
c_{n, i}^{\prime}=c_{n, i} \cosh \left(\theta_{n, i}\right) \quad \tilde{c}_{n, i}^{\prime}=c_{n, i} \sinh \left(\theta_{n, i}\right)
$$

with

$$
\theta_{n, i}=\operatorname{arctanh}\left(\exp \left\{-\frac{\omega_{n, i}}{2 k_{\mathrm{B}} T}\right\}\right)
$$

Figure $3 b, c$ illustrates the thermalized component spectral densities $^{39,40}$ defined by the vibronic couplings of eq 13 . On the basis of these thermalized SDs, the number of low-frequency modes included in the TFD treatment could have been slightly augmented.

The vibronic Hamiltonian of eq 7, restricted to the acceptor modes, can thus be extended using the TFD approach to give

$$
\begin{aligned}
& H_{T}^{\mathrm{ph}}+H_{T}^{\mathrm{el}-\mathrm{ph}}=\sum_{n=0}^{24}\left(\sum _ { j = 6 } ^ { 3 0 } \left[\frac{1}{2} \omega_{n, j}^{\mathrm{A}}\left(\left(p_{n, j}^{\mathrm{A}}\right)^{2}+\left(q_{n, j}^{\mathrm{A}}\right)^{2}\right)\right.\right. \\
& \left.+c_{n, j}^{\mathrm{A}} q_{n, j}^{\mathrm{A}}|n\rangle\langle n|\right]+\sum_{j=1}^{5}\left[\frac{1}{2} \omega_{n, j}^{\mathrm{A}}\left(\left(p_{n, j}^{\mathrm{A}}\right)^{2}+\left(q_{n, j}^{\mathrm{A}}\right)^{2}\right)+c_{n, j}^{\mathrm{A}} q_{n, j}^{\mathrm{A}}|n\rangle\langle n|\right] \\
& \left.+\sum_{j=1}^{5}\left[-\frac{1}{2} \omega_{n, j}^{\mathrm{A}}\left(\left(\tilde{p}_{n, j}^{\mathrm{A}}\right)^{2}+\left(\tilde{q}_{n, j}^{\mathrm{A}}\right)^{2}\right)+\tilde{c}_{n, j}^{\mathrm{A}^{\prime}} \tilde{q}_{n, j}^{\mathrm{A}}|n\rangle\langle n|\right]\right)
\end{aligned}
$$

where the zeroth-order part of the "tildian", $\tilde{H}_{T}^{\mathrm{ph}}$, was chosen of the same form as $H_{T}^{\mathrm{ph}}$ (i.e., $\left.\tilde{H}_{T}^{\mathrm{ph}}=\sum_{j=1}^{5} \frac{1}{2} \omega_{n, j}^{\mathrm{A}}\left(\left(\tilde{p}_{n, j}^{\mathrm{A}}\right)^{2}+\left(\tilde{q}_{n, j}^{\mathrm{A}}\right)^{2}\right)\right)$, leading to invariance under the Bogoliubov transformation of eq 12..$^{37,39-41}$ The full TFD Hamiltonian of eq 15 consists of a total of 875 vibrational modes (i.e., 750 physical modes and 125 tilde modes) which will be included in the quantum-dynamical simulations.

3.3. Quantum Dynamical Simulations. In order to carry out accurate quantum-dynamical simulations for a system of 25 electronic states and 875 vibrational modes, we employ the highly efficient multilayer version (ML-MCTDH) $)^{42-45}$ of the

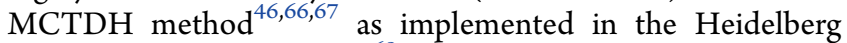
MCTDH program package. ${ }^{68}$ As mentioned above, calculations are carried out at the wave function level within the extended Hilbert space defined by the TFD approach.

A harmonic oscillator Discrete Variable Representation (DVR) was chosen as primitive basis for all phonon modes, with 20 basis functions for all modes. The structure of the multilayer tree (see Figure 4), with 8 layers and mode combinations of one or two modes in the lowest layer, was kept the same for all simulations; the number of single particle functions (SPFs) was also kept unchanged and was adapted to the calculation at the highest temperature, which exhibits the highest degree of correlation. In the hierarchical tree, the thermalized low-frequency modes (five modes per PDI fragment and their tilde analogs) were arranged into a separate branch, complementary to the remaining modes (25 modes per PDI fragment). Among the low-frequency modes, physical and tilde modes were grouped together in pairs in the lowest layer. For all modes, the lower layers were constructed such as to match the fragment structure of the Hamiltonian. Natural-orbital populations were considered reasonably converged with a threshold of about $1 \%$. (As a rule of thumb, this is the threshold considered in typical MCTDH calculations; however, convergence may also depend on the structure of the multilayer-tree. $)^{44}$ Norm conservation was obeyed up to $10^{-6}$. The simulations were carried out within an interval of $2.5 \mathrm{ps}$ for temperatures of $0 \mathrm{~K} \mathrm{up}$ to $300 \mathrm{~K}$ in steps of $50 \mathrm{~K}$ and fields of $0,1 \times 10^{6}, 5 \times 10^{6}, 10 \times$
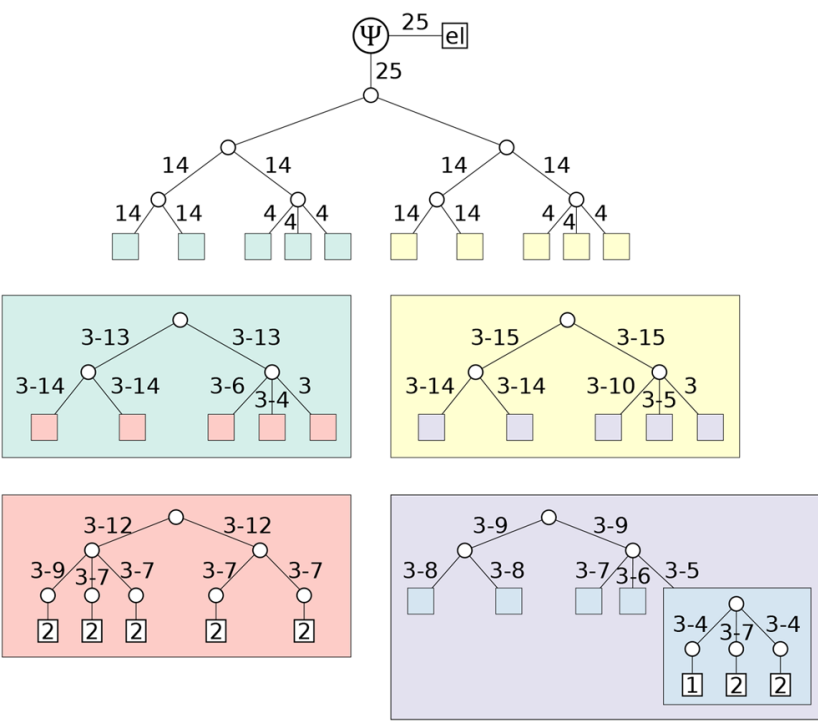

Figure 4. Multilayer tree employed in the ML-MCTDH calculations described in the main text. The hierarchical tree structure comprises $M$ $=8$ layers and describes the thermofield wave function for 25 electronic states and 875 modes. The electronic branch (labeled "el") is taken as a separate particle, in line with the so-called single-set formulation employed in standard ML-MCTDH. ${ }^{68}$ Circles represent nodes, colored rectangles represent portions of the tree structures that are used repeatedly and shown in detail further below, and small white rectangles indicate the particles in the final layer where the number shows how many modes are combined into a single particle. Numbers next to lines connecting two nodes are the number of associated SPFs. The branch comprising the green and red boxes (l.h.s.) represents the set of TFD low-frequency modes (i.e., five physical modes and five tilde modes per PDI fragment), while the branch comprising the yellow and violet boxes (r.h.s.) comprises all remaining modes (i.e., 25 modes per PDI fragment). Each red or violet box contains modes belonging to a given PDI fragment. The number of SPFs varies between equivalent subbranches, with the lowest and highest numbers indicated.

$10^{6}, 15 \times 10^{6}$, and $20 \times 10^{6} \mathrm{~V} \mathrm{~m}^{-1}$. For each run with a propagation time of $2.5 \mathrm{ps}$, CPU times for the present calculations lie around $210 \mathrm{~h}$ on a $2.6 \mathrm{GHz}$ Xeon E5-2690v4 processor (single-thread).

3.4. Rate Computation. Since charge separation in the DA lattice under study is comparatively slow and can be characterized as an escape process from the bound domain of an effective $\mathrm{e}-\mathrm{h}$ potential, we will use the quantum dynamical results in conjunction with an approximate rate theory using the flux-over-population method. ${ }^{33-35}$ Here, the escape rate is given as

$$
k=\frac{j\left(n_{\mathrm{e}}\right)}{P_{\text {bound }}^{0}}
$$

where $P_{\text {bound }}^{0}$ is the population of the initial bound species ( $\left.P_{\text {bound }}^{0} \simeq 1\right)$ and $j\left(n_{\mathrm{e}}\right)$ is the flux at the lattice point $n_{\mathrm{e}}$ marking the escape threshold (i.e., separating the bound and free $\mathrm{e}-\mathrm{h}$ species). The flux-over-population approach is adapted to a quasi-stationary nonequilibrium state ${ }^{35}$ where $P_{\text {bound }}(t) \sim$ constant, noting that this condition is only approximately fulfilled in the present simulations which do not use open boundary conditions. However, the charge separation is slow enough to use the approximation $P_{\text {bound }}^{0} \simeq 1$ and neglect reflection effects from the boundaries of the finite-dimensional 
lattice on the time scale of observation. The applicability of the method in the present context is justified (i) if the domains representing bound versus free $\mathrm{e}-\mathrm{h}$ pairs can be spatially separated at $n=n_{\mathrm{e}}$, at least in an approximate fashion and (ii) if the resulting flux $j\left(n_{\mathrm{e}}\right)$ toward the charge-separated domain is approximately constant.

More generally, an instantaneous escape rate can be introduced $^{69,70}$

$$
k(t)=\frac{j\left(n_{\mathrm{e}}, t\right)}{P_{\text {bound }}(t)}
$$

which yields the time average

$$
\bar{k}=\lim _{t \rightarrow \infty} \frac{1}{t} \int_{0}^{t} \mathrm{~d} t k(t)
$$

In our study, though, we use the simpler time-independent framework of eq 16 since we observe a rate type behavior, with nearly constant $k$, if a suitable partitioning into bound and free domains is chosen. Therefore, time-averaging is only employed over short propagation intervals to average over vibrationinduced fluctuations. In the above expressions, the flux is defined by a continuity equation

$$
j\left(n_{\mathrm{e}}, t\right)=-\frac{\mathrm{d}}{\mathrm{d} t} \sum_{n=n_{0}}^{n_{\mathrm{e}}} P(n, t)=-\dot{P}_{\text {bound }}
$$

To compute the flux that is required in the above rate equations, time-dependent state populations are obtained from quantum dynamical simulations for the full electron-phonon system, as described above:

$$
P(n, t)=\operatorname{Tr}_{\mathrm{vib}}\{|n\rangle\langle n \mid \psi(t)\rangle\langle\psi(t)|\}
$$

where $|\psi(t)\rangle$ refers to the time-evolving electronic-vibrational wave function and $|n\rangle$ refers to $\mathrm{e}-\mathrm{h}$ states specifying the electron position at lattice site $n$ while the hole position is fixed.

A more detailed analysis would involve the computation of flux correlation functions that directly relate to the rate. ${ }^{35}$ The simpler flux-over-population method described above is appropriate when the process is rapid enough to permit a direct computation of the free species $\left(1-P_{\text {bound }}(t)\right)$. Within this framework, a unique rate according to eq 18 is obtained if the average of $k(t)$ over the propagation time scale agrees with the long-time average of eq $18 .^{35,62,69}$ This is the case if the flux reaches its asymptotic limit within the propagation time. For short propagation times, as presented in the present work, this criterion cannot be rigorously verified.

\section{RESULTS}

Here, we report on the results of our quantum dynamical study and rate computation. In section 4.1, vibrationally averaged electronic eigenstates are shown to obtain insight into the energetics and delocalization effects. Following this, section 4.2 comments on the initial conditions for the dynamical calculations and section 4.3 reports on the quantum dynamical results and rate computation.

4.1. Vibrationally Averaged Electronic Eigenstates. To understand the charge separation process, it is useful to consider vibrationally averaged electronic eigenstates of the e-h system. These are defined as the eigenstates of an effective timedependent Hamiltonian restricted to the electronic degrees of freedom only, obtained upon tracing over the vibrational degrees of freedom:

$$
H_{\text {eff }}^{\mathrm{el}}(t)=T r_{\text {vib }}\left\{\left(H^{\mathrm{el}}+H^{\mathrm{field}}+H^{\mathrm{el}-\mathrm{ph}}\right)|\psi(t)\rangle\langle\psi(t)|\right\}
$$

As discussed below (see also Supporting Information section S4), these eigenstates are similar to the pure electronic eigenstates (i.e., eigenstates of $\left(H^{\mathrm{el}}+H^{\text {field }}\right)$ in the absence of the averaged electron-phonon interaction), but slight modifications occur in the delocalization pattern and also in the eigenenergies, mainly due to trapping (polaronic) effects.

Figure $2 \mathrm{~b}-\mathrm{e}$ shows these effective electronic states (i.e., eigenstates of $\left.H_{\text {eff }}^{\mathrm{el}}(t)\right)$ and their energies $\mathcal{E}_{n}$ for the field $E=2 \times$ $10^{7} \mathrm{Vm}^{-1}$ and different times (i.e., $t=0,10$, and $300 \mathrm{fs}$ ), along with a time average. For reference, a representation of the effective barrier $\left\{\varepsilon_{n}\right\}$ according to eq 4 is shown in Figure 2a). At $t=0 \mathrm{fs}$, the eigenstates of $H_{\mathrm{eff}}^{\mathrm{el}}(t=0)$ are identical to the pure electronic eigenstates, due to the lack of electronic-vibrational correlations in the initial state. The next snapshot, at $t=10 \mathrm{fs}$, reveals that a polaronic stabilization of the bound $\mathrm{e}-\mathrm{h}$ state of around $0.15 \mathrm{eV}$ arises, which increases the effective Coulomb barrier as compared with the pure electronic eigenstate spectrum. By the same token, the electronic mixing between quasi-bound and dissociative $\mathrm{e}-\mathrm{h}$ states is reduced. This is counteracted to some extent by delocalization effects which tend to reduce the effective barrier height. From about $t=100 \mathrm{fs}$ onward, the eigenvector structure remains essentially unchanged (i.e., the system has reached a quasi-stationary state which is characterized by partially delocalized electronic eigenstates (see Movie S1)). The effective barrier height is around $0.4 \mathrm{eV}$ (i.e., about twice as large as the reorganization energy of an individual PDI fragment).

From Figure $2 \mathrm{~b}-\mathrm{e}$, it is seen that the lowest-energy eigenstates in the range between -0.75 and $-0.35 \mathrm{eV}$ are typically delocalized across two or three sites and form a staircase-like pattern. At higher field strengths like $E=2 \times 10^{7}$ $\mathrm{Vm}^{-1}$ as illustrated here, the states around $-0.35 \mathrm{eV}$ become more delocalized, comprising around five sites. These states include near-degenerate prebarrier versus postbarrier states that can give rise to an effective tunneling through the Coulomb barrier. ${ }^{14,71}$ The higher-lying states (above $-0.35 \mathrm{eV}$ ) are more diffuse and represent delocalized barrier states. We expect that these states will not play a significant role in the thermally activated charge separation process.

From the eigenvector representation, the definition of a unique separation between the bound and free domains, separated at a critical site value $n_{\mathrm{e}}$ permitting escape toward larger $\mathrm{e}-\mathrm{h}$ separations, is not entirely obvious. This is all the more so the case for smaller fields that do not resemble an effective saddle-point potential (see Supporting Information section S4). However, the above picture shows that the lowlying $\mathrm{e}-\mathrm{h}$ states beyond $n_{\mathrm{e}}=9$ and 10 have negligible contributions in the bound region and can therefore be assumed to be prone to dissociation. Therefore, we identify these values as a suitable threshold for the rate analysis described above. In practice, averages over rates computed for $n_{\mathrm{e}}=9$ and 10 will be taken.

4.2. Initial Conditions. In the present simulations, the initial condition corresponds to the charge separated state $\operatorname{CS}(0)=|0\rangle=\left|\nu_{\mathrm{e}}=0, \eta_{\mathrm{h}}=0\right\rangle$ where both electron and hole are located on a given covalently bound DA dyad (i.e., the "CT state"). This state is generated by an exciton dissociation event, starting from an initial photogenerated excitonic species localized on the D moiety, which is not explicitly included in the present simulations. Exciton dissociation likely happens 

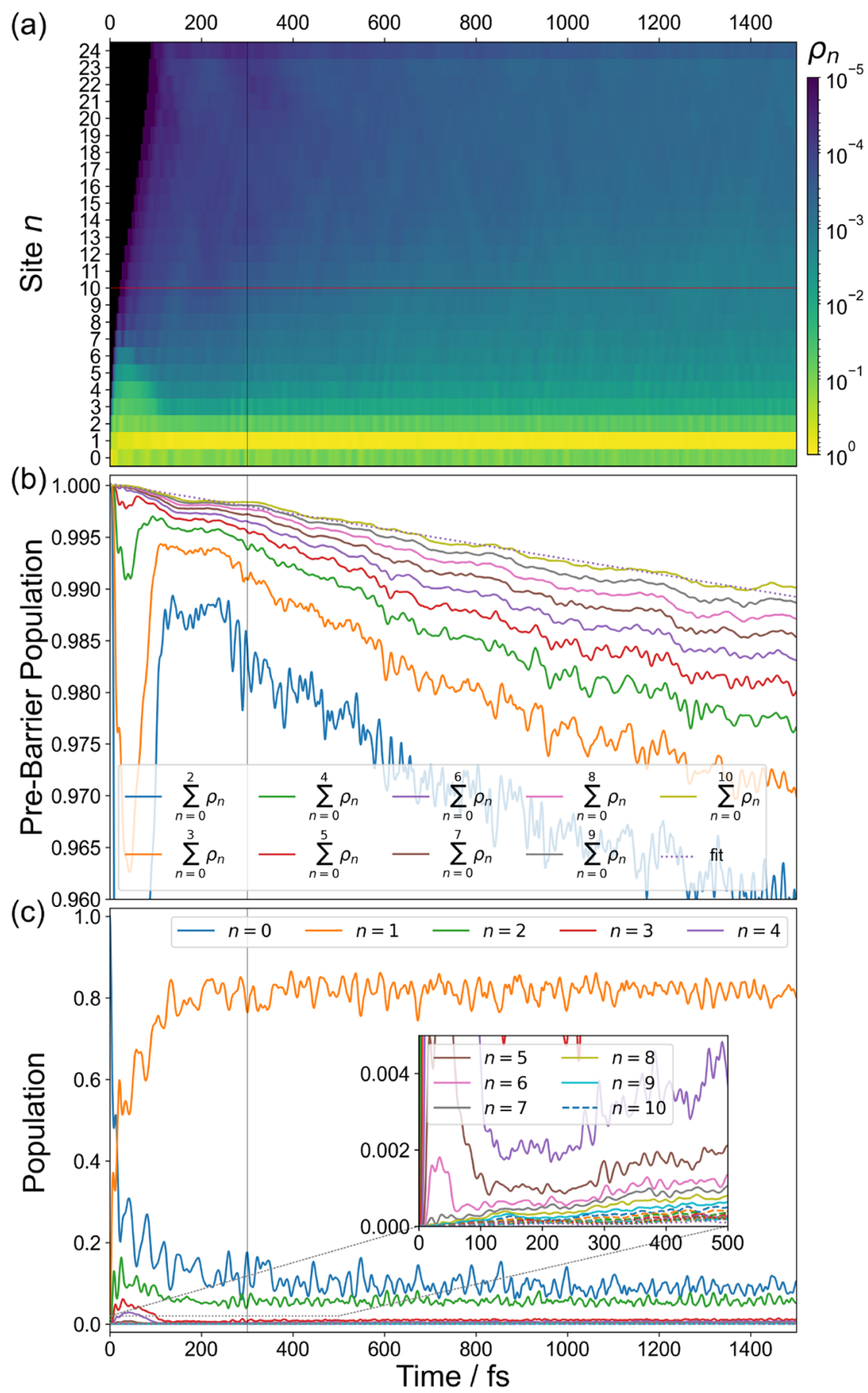

Figure 5. (a) Density plot illustrating the populations of the $\mathrm{e}-\mathrm{h}$ states as a function of time during a propagation interval of $1.5 \mathrm{ps}$, at $T=0 \mathrm{~K}$ and $E=5$ $\times 10^{6} \mathrm{Vm}^{-1}$. The populations, reported on a logarithmic scale, remain to a large extent confined in the bound region of the effective e-h potential, below the red line at $n_{\mathrm{e}}=10$. The dissociative portion of the population, after $1.5 \mathrm{ps}$, is of the order of $10^{-3}$, as can be seen in the upper part of the figure. (b) Integrated $\mathrm{e}-\mathrm{h}$ state populations for different choices of the barrier site $n_{\mathrm{e}}$, again at $T=0 \mathrm{~K}$ and $E=5 \times 10^{6} \mathrm{Vm}^{-1}$. The dashed line marks an approximately linear decay for $n_{\mathrm{e}}=10$. (c) Time-evolving state populations for $n=0, \ldots, 10$, highlighting coherent dynamics in the bound domain during the first $300 \mathrm{fs}$ (marked by a black vertical line), followed by a quasi-stationary regime. The latter is characterized by a rate-like, slow increase in population across the effective Coulomb barrier, as indicated in the inset.

within tens or hundreds of picoseconds in the present system, following our earlier analysis of the same dyad system in solution phase. ${ }^{31}$ As summarized in section 2, among various chemically modified DA dyad realizations analyzed in ref 31 , the present system corresponds to the $\mathrm{D}_{0} \delta_{+} \mathrm{A}$ species which belongs to a DA class that yield a charge separated state within $\sim 100$ ps in solution. In preliminary spectroscopic investigations of the
$\mathrm{D}_{0} \delta_{+} \mathrm{A}$ species in a thin film, ${ }^{32}$ it was found that the initial exciton is likely of $H$ aggregate type (i.e., delocalized across several D species), but charge separation remains similarly slow as in solution phase. Therefore, the charge separated CS(0) type species is likely not formed in a delocalized coherent superposition states. Furthermore, due to the comparatively slow time scale of formation of the charge separated species, the 


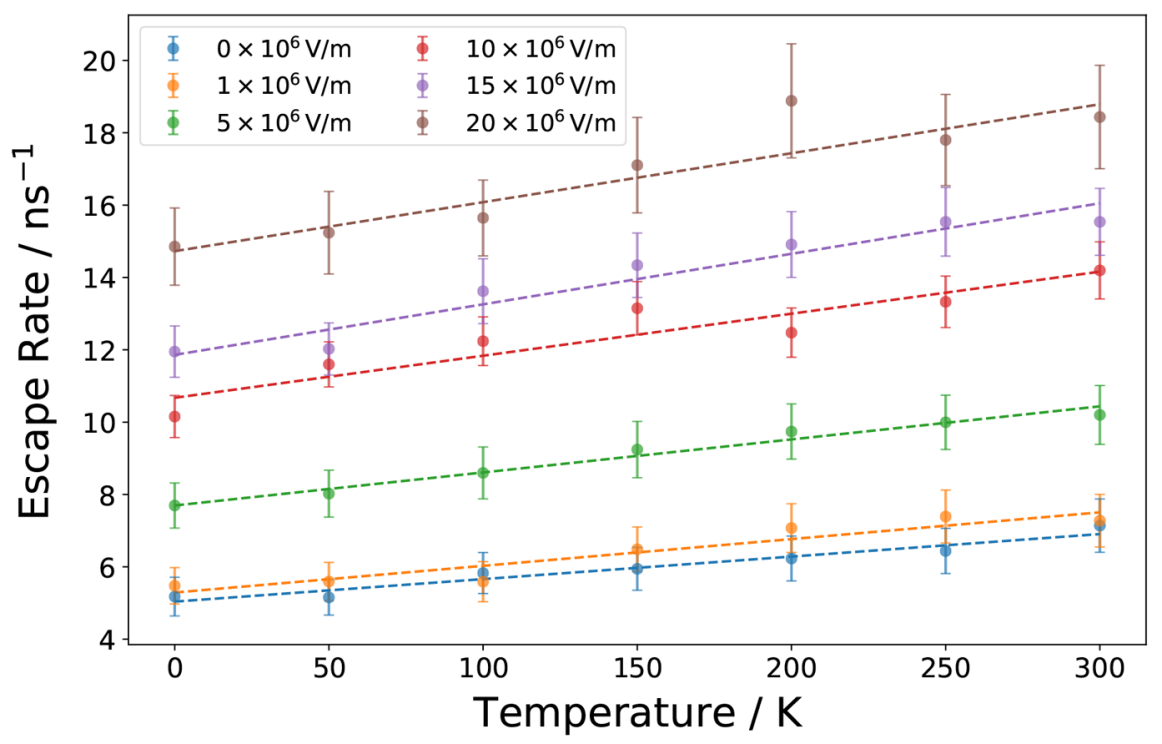

Figure 6. Escape rates $k$ (i.e., $\mathrm{e}-\mathrm{h}$ dissociation rates) computed from the time evolution according to eq 16, showing that the rates show a moderate increase with both temperature and field strength. The error bars indicate standard deviations computed by sampling over variable linear fit intervals as described in the text. The dashed lines, corresponding to a linear fit, are a guide for the eye.

initial state carries little vibronic excess energy that could facilitate passage across the Coulomb barrier.

These factors all taken together, the initial condition is defined as a localized low-energy interfacial charge-separated state $(\mathrm{CS}(0))$ residing on a given DA dyad. We therefore expect that rapid mixing occurs with the $\operatorname{CS}(1)$ state which is neardegenerate with the $\operatorname{CS}(0)$ state, but other than that, $\mathrm{e}-\mathrm{h}$ separation is a thermally activated, slow process, excluding ultrafast long-range $\mathrm{e}-\mathrm{h}$ separation. Yet, delocalization effects could play a non-negligible role in promoting the coupling between the bound and dissociative $\mathrm{e}-\mathrm{h}$ domains.

4.3. Time-Evolving State Populations and Rate Computation. ML-MCTDH calculations as described above were carried out for different temperatures and field strengths. The aim of these calculations is to use a full quantum dynamical setting in order to explore the role of coherent transients, along with the emergence of a kinetic description, which can be characterized by approximate escape rates as described in section 3.4. In the calculations reported below, conditions were varied from $T=0 \mathrm{~K}$ to $T=300 \mathrm{~K}$ and field-free conditions up to $E=2 \times 10^{7} \mathrm{Vm}^{-1}$, for a propagation time of $2.5 \mathrm{ps}$. For reference, experiments for the present system were carried out at room temperature and a field strength of $5 \times 10^{6} \mathrm{Vm}^{-1}$. ${ }^{29}$

Since the charge separation process is slow, the integrated postbarrier population $\left(1-P_{\text {bound }}\right)$ for $n>9$ is found in the range of $10^{-3}$, as can be seen from Figure $5 \mathrm{a}$ ). In a complementary fashion, the integrated bound population for different cutoff values $n_{\mathrm{e}}$ is shown in Figure $5 b$ ). For $n_{\mathrm{e}} \geq 9$, it is seen that the decay is approximately linear, suggesting that a kinetic interpretation of the process in terms of transfer between the bound and dissociative domains is appropriate. According to eqs 16 and 17 , the $\mathrm{e}-\mathrm{h}$ dissociation rate ("escape rate") $k$ is approximated by this linear population decay from the bound region (i.e., $\dot{P}_{\text {bound }}$ in eq 19). Meanwhile, the population in the bound domain, shown in Figure 5c) undergoes marked transient effects up to $t \sim 300 \mathrm{fs}$, involving the onset of trapping and a coherent exchange of population between the CS(0) and CS(1) states. From $t \sim 300$ fs onward, the coherent transients have subsided and the populations are found in a quasi-stationary state where vibronic oscillations persist.

The threshold values $n_{\mathrm{e}}=9$ and 10 are found to be appropriate as boundary between the quasi-bound $\mathrm{e}-\mathrm{h}$ states and dissociative $\mathrm{e}-\mathrm{h}$ states, as suggested by the eigenstate structure described in section 4.1. The same threshold also holds approximately for other field strengths, such that we refer to averaged results obtained for these values to compute rates according to eqs 16 and 17.

Using this procedure, the temperature- and field-dependent rates reported in Figure 6 are obtained in the absence of static disorder. Results were averaged for $n_{\mathrm{e}}=9$ and 10, and over variable linear fit intervals ( 300 fs to $1 \mathrm{ps}$, and $300 \mathrm{fs}$ to $1.5 \mathrm{ps}$ ); standard deviations were computed from this comparison. As further discussed in the Supporting Information section S5, the integral over the instantaneous escape rate $k(t)=$ $-\dot{P}_{\text {bound }} / P_{\text {bound }}(t)$ (see eqs 17 and 18), computed during the propagation time $(2.5 \mathrm{ps})$ gives similar values. Even though fluctuations in the present system are too large to ascertain from the instantaneous rates $k(t)$ that a constant value has been reached (see the discussion in section 3.4), the near-linear decay illustrated in Figure $5 \mathrm{~b}$ is clearly indicative of a short-time rate behavior, $P_{\text {bound }}(t) \simeq 1-k t$. Whether the rates obtained from the present short-time propagation require modification when eq 18 is computed for longer time scales, calls for further analysis by complementary methods (e.g., master equation approaches). ${ }^{72,73}$

It is seen that the rates range from about 5 to $20 \mathrm{~ns}^{-1}$ and increase with temperature and field strength as expected. The rates are non-negligible at $T=0 \mathrm{~K}$ and exhibit a moderate, approximately linear temperature dependence, with an increase of the rate around $30 \%$ between $T=0 \mathrm{~K}$ and $T=300 \mathrm{~K}$. The finite rates at $T=0 \mathrm{~K}$ are a nonclassical feature that is entirely due to the "quantum fluctuations" of the explicit phonon distribution. The field dependence is found to be monotonic, as expected, and the rates approximately triple between the fieldfree case and a field of $2 \times 10^{7} \mathrm{~V} / \mathrm{m}$.

Finally, we also employed the simulation results to compute approximate mobilities in the region where the $\mathrm{e}-\mathrm{h}$ pair has 
become unbound (i.e., for $n>n_{\mathrm{e}}$ ). To this end, the mean carrier displacement distance

$$
\langle D\rangle(t)=\sum_{n>n_{\mathrm{e}}} D(n) P(n, t)
$$

was computed, where $D(n)$ is the site-dependent $\mathrm{e}-\mathrm{h}$ distance and $P(n, t)$ are time-dependent state populations according to eq 20. From this, the mean velocity $\langle v\rangle=\mathrm{d}\langle D\rangle / \mathrm{d} t$ is obtained, yielding the mobility $\mu=\langle v\rangle / E$. The resulting mobility values are of the order of $5 \times 10^{-2} \mathrm{~cm}^{2} /(\mathrm{Vs})$. Even though this estimate should be considered as approximate, due to the limited lattice size and the absence of intermolecular modes in the present study, it is compatible with the experimentally observed mobility of $\mu_{\mathrm{e}}=0.02 \mathrm{~cm}^{2} /(\mathrm{V} \mathrm{s}) .^{32}$ Such comparatively large mobility values have also been observed for related ordered PDI assemblies. ${ }^{9,74}$

\section{DISCUSSION AND CONCLUSIONS}

The present simulations involve no free parameters and accurately account for the influence of vibronic effects, field strength, and temperature on the charge separation dynamics of the full DA system. The underlying microelectrostatic computations give a precise description of the local electrostatic environment. An assumption built into the present description is that intermolecular phonons do not play an important role, which is compatible with the moderate mobility values which suggest a hopping picture ruled by intramolecular relaxation, rather than band-like phenomena. A further assumption, inherent in the microelectrostatic treatment, is that the (electronic) polarization response of the dielectric environment to the moving charge is instantaneous, leading to an equilibrium treatment. Nuclear rearrangements leading to polarization effects (i.e., reorientational phonon motions) can be disregarded, given the crystal-like, mesomorphic structure of the DA assembly. All aspects taken together, the present description provides a faithful, fully microscopic picture of the $\mathrm{e}-\mathrm{h}$ separation process.

In the present analysis, a kinetic rate picture emerges from wave function propagation for the full electron-phonon system, by identifying a separation between bound and dissociative $\mathrm{e}-\mathrm{h}$ domains, such that a near-constant flux toward the latter is observed. Hence, the $\mathrm{e}-\mathrm{h}$ dissociation rate can be described by a constant escape rate from the bound domain, using a flux-overpopulation approach. As shown in the above discussion, the electronic eigenstate spectrum determines the effective separation line between bound and free $\mathrm{e}-\mathrm{h}$ species. While the $\mathrm{e}-\mathrm{h}$ eigenstate structure and the associated delocalization pattern are slightly modified under the effect of vibrational averagingespecially due to trapping effects-the boundary between the domains remains essentially intact. However, this boundary is more arbitrary than that in a typical saddle-point potential, due to the nature of the molecular aggregate system. Even so, the near-linear population decay out of the bound domain shows the validity of the rate perspective.

As for the role of electronic delocalization in promoting $\mathrm{e}-\mathrm{h}$ separation, ${ }^{11,15}$ this aspect does play a role to some extent in the present system. In general, $\mathrm{e}-\mathrm{h}$ dissociation is enhanced by the fact that transitions take place between partially delocalized electronic eigenstates, leading to a large increase in the effective $\mathrm{e}-\mathrm{h}$ distance for a given transition. However, electronic eigenstate delocalization is typically restricted to $2-3$ PDI units in the bound domain, in line with experimental observations. ${ }^{17,18}$ In the dissociative domain, more pronounced delocalization effects occur (around five units), especially at larger field strengths, yet the effective $\mathrm{e}-\mathrm{h}$ binding energy remains large, on the order of $0.4 \mathrm{eV}$, such that the dissociation process is comparatively slow. Notwithstanding, free carrier formation can be highly efficient. ${ }^{10,21}$

From the above vantage point, the transition between the bound and dissociative $\mathrm{e}-\mathrm{h}$ states is driven by vibronic coupling (i.e., dynamic disorder). That is, phonon modes are expected to induce multiple nonadiabatic crossing events which couple bound and dissociative $\mathrm{e}-\mathrm{h}$ states. These dynamical events are treated explicitly and fully quantum mechanically in the present setup. This permits a direct observation of a transition between a short-time coherent regime (here, around $300 \mathrm{fs}$ ) and a quasistationary regime at longer times which gives rise to the $\mathrm{e}-\mathrm{h}$ dissociation kinetics. Given the strength of vibronic interactions and the inclusion of "quantum fluctuations" in our simulations, transitions to the high-energy dissociative $\mathrm{e}-\mathrm{h}$ states are found to take place even at $T=0 \mathrm{~K}$, different from the prediction of classical rate models. Similar findings have been reported for simpler 1D models with one effective high-frequency vibration per molecule. $^{75,76}$ The temperature dependence observed in our simulations is near-linear, with a $\sim 30 \%$ increase between $0 \mathrm{~K}$ and $300 \mathrm{~K}$. These trends are compatible with various observations where a minor or negligible temperature dependence was observed for $\mathrm{e}-\mathrm{h}$ separation in various materials, ${ }^{10,11,77,78}$ including PDI-based nanoribbon architectures. ${ }^{49}$

One of the goals of the present analysis is to provide benchmark results for the description of $\mathrm{e}-\mathrm{h}$ dissociation rates by kinetic Monte Carlo simulations for the same system, modeled for a much larger, 2D lattice in conjunction with a computed 2D microelectrostatic map. These results will be reported in a follow-up publication.

While experimentally measured $\mathrm{e}-\mathrm{h}$ separation rates are not available for the present system, the resulting rates of the order of $5-20 \mathrm{~ns}^{-1}$ are plausible in the absence of static disorder. Preliminary time-resolved experiments conducted on the present system $^{32}$ showed decay features with rate components of $\sim 0.2$ and $\sim 10 \mathrm{~ns}^{-1}$ but revealed that competing processes may occur that are not accounted for in our model. In particular, geminate recombination ${ }^{31,50}$ and excimer formation ${ }^{19,79,80}$ have been discussed as a source of loss pathways in PDI-based systems. The present Hamiltonian approach does not yet include these processes, but it is entirely suitable to extend the analysis to accommodate various molecular mechanisms that modify $\mathrm{e}-\mathrm{h}$ separation in realistic systems.

\section{ASSOCIATED CONTENT}

\section{(s) Supporting Information}

The Supporting Information is available free of charge at https://pubs.acs.org/doi/10.1021/acs.jpcc.1c06374.

Details regarding the construction of an effective 1D Coulomb potential based on microelectrostatic data, the computation of transfer integrals, spectral density computations, the electronic eigenstate analysis, details of the rate computation (PDF)

Movie S1: movie illustrating the effective eigenvector evolution complementary to Figure 2 (MP4)

Relevant vibrational frequencies and vibronic couplings (PDF) 


\section{AUTHOR INFORMATION}

\section{Corresponding Author}

Irene Burghardt - Institute for Physical and Theoretical Chemistry, Goethe University, 60438 Frankfurt/Main, Germany; ๑ orcid.org/0000-0002-9727-9049; Email: burghardt@chemie.uni-frankfurt.de

\section{Authors}

Dominik Brey - Institute for Physical and Theoretical Chemistry, Goethe University, 60438 Frankfurt/Main, Germany

Wjatscheslaw Popp - Institute for Physical and Theoretical Chemistry, Goethe University, 60438 Frankfurt/Main, Germany

Praveen Budakoti - Institute for Physical and Theoretical Chemistry, Goethe University, 60438 Frankfurt/Main, Germany

Gabriele D'Avino - Grenoble Alpes University, CNRS, Grenoble INP, Institut Néel, 38042 Grenoble, France; (-) orcid.org/0000-0002-5897-2924

Complete contact information is available at: https://pubs.acs.org/10.1021/acs.jpcc.1c06374

\section{Notes}

The authors declare no competing financial interest.

\section{ACKNOWLEDGMENTS}

We gratefully acknowledge discussions with S. Méry, S. Haacke, R. Hegger, K. Schwinn, R. Martinazzo, and R. Binder. S. Méry and M. Brinkmann are further acknowledged for providing the ED structure of the donor-acceptor system under study. Funding for this project was granted by the Deutsche Forschungsgemeinschaft (DFG) in the framework of the project MolNanoMat (BU-1032-2).

\section{REFERENCES}

(1) Onsager, L. Initial Recombination of Ions. Phys. Rev. 1938, 54, 554.

(2) Braun, C. Electric field assisted dissociation of charge transfer states as a mechanism of photocarrier production. J. Chem. Phys. 1984, $80,4157$.

(3) Wojcik, M.; Tachiya, M. Accuracies of the empirical theories of the escape probability based on Eigen model and Braun model compared with the exact extension of Onsager theory. J. Chem. Phys. 2009, 130, 104107.

(4) Arkhipov, V. I.; Heremans, P.; Bässler, H. Why is exciton dissociation so efficient at the interface between a conjugated polymer and an electron acceptor? Appl. Phys. Lett. 2003, 82, 4605.

(5) Nenashev, A. V.; Baranovskii, S. D.; Wiemer, M.; Jansson, F.; Österbacka, R.; Dvurechenskii, A. V.; Gebhard, F. Theory of exciton dissociation at the interface between a conjugated polymer and an electron acceptor. Phys. Rev. B: Condens. Matter Mater. Phys. 2011, 84, 035210.

(6) Baranovskii, S. D.; Wiemer, M.; Nenashev, A. V.; Jansson, F.; Gebhard, F. Calculating the Efficiency of Exciton Dissociation at the Interface between a Conjugated Polymer and an Electron Acceptor. J. Phys. Chem. Lett. 2012, 3, 1214.

(7) Bortz, A. B.; Kalos, M. H.; Lebowitz, J. L. A new algorithm for Monte Carlo simulation of Ising spin systems. J. Comput. Phys. 1975, 17, 10.

(8) Deibel, C.; Strobel, T.; Dyakonov, V. Origin of the Efficient Polaron-Pair Dissociation in Polymer-Fullerene Blends. Phys. Rev. Lett. 2009, 103, 036402.
(9) Lin, L.; Geng, H.; Shuai, Z.; Luo, Y. Theoretical insights into the charge transport in perylene diimides based n-type organic semiconductors. Org. Electron. 2012, 13, 2763.

(10) Köhler, A.; Bässler, H. "Hot or cold": how do charge transfer states at the donor-acceptor interface of an organic solar cell dissociate? Phys. Chem. Chem. Phys. 2015, 17, 28451.

(11) Athanasopoulos, S.; Schauer, F.; Nádaždy, V.; Weiß, M.; Kahle, F.; Scherf, U.; Bässler, H.; Köhler, A. What is the Binding Energy of a Charge Transfer State in an Organic Solar Cell? Adv. Energy Mater. 2019, 9, 1900814.

(12) Gélinas, S.; Rao, A.; Kumar, A.; Smith, S. L.; Chin, A. W.; Clark, J.; van der Poll, T. S.; Bazan, G. C.; Friend, R. H. Ultrafast Long-Range Charge Separation in Organic Semiconductor Photovoltaic Diodes. Science 2014, 343, 512.

(13) Provencher, F.; Bérubé, N.; Parker, A.; Greetham, G. M.; Towrie, M.; Hellmann, C.; Cote, M.; Stingelin, N.; Silva, C.; Hayes, S. Direct observation of ultrafast long-range charge separation at polymerfullerene heterojunctions. Nat. Commun. 2014, 5, 4288.

(14) Tamura, H.; Burghardt, I. Ultrafast charge separation in organic photovoltaics enhanced by charge delocalization and vibronically hot exciton dissociation. J. Am. Chem. Soc. 2013, 135, 16364.

(15) Pace, N. A.; Reid, O. G.; Rumbles, G. Delocalization Drives Free Charge Generation in Conjugated Polymer Films. ACS Energy Lett. 2018, 3, 735 .

(16) Feier, H. M.; Reid, O. G.; Pace, N. A.; Park, J.; Bergkamp, J. J.; Sellinger, A.; Gust, D.; Rumbles, G. Local Intermolecular Order Controls Photoinduced Charge Separation at Donor/Acceptor Interfaces in Organic Semiconductors. Adv. Energy Mater. 2016, 6, 1502176.

(17) Hartnett, P. E.; Mauck, C. M.; Harris, M. A.; Young, R. M.; Wu, Y.; Marks, T. J.; Wasielewski, M. R. Influence of Anion Delocalization on Electron Transfer in a Covalent Porphyrin Donor-Perylenediimide Dimer Acceptor System. J. Am. Chem. Soc. 2017, 139, 749.

(18) Alzola, J. M.; Powers-Riggs, N. E.; La Porte, N. T.; Young, R. M.; Marks, T. J.; Wasielewski, M. R. Photoinduced electron transfer from zinc meso-tetraphenylporphyrin to a one-dimensional perylenediimide aggregate: Probing anion delocalization effects. J. Porphyrins Phthalocyanines 2020, 24, 143.

(19) Würthner, F.; Saha-Möller, C. R.; Fimmel, B.; Ogi, S.; Leowanawat, P.; Schmidt, D. Perylene Bisimide Dye Assemblies as Archetype Functional Supramolecular Materials. Chem. Rev. 2016, 116, 962.

(20) Vithanage, D. A.; Devizis, A.; Abramavicius, V.; Infahsaeng, Y.; Abramavicius, D.; MacKenzie, R.; Keivanidis, P. E.; Yartsev, A.; Hertel, D.; Nelson, J.; Sundström, V.; Gulbinas, V. Visualizing charge separation in bulk heterojunction organic solar cells. Nat. Commun. 2013, 4, 2334.

(21) Vandewal, K.; et al. Efficient charge generation by relaxed chargetransfer states at organic interfaces. Nat. Mater. 2014, 13, 63.

(22) Popp, W.; Brey, D.; Binder, R.; Burghardt, I. Quantum Dynamics of Exciton Transport and Dissociation in Multichromophoric Systems. Annu. Rev. Phys. Chem. 2021, 72, 591.

(23) Lee, Y.; Gomez, E. D. Challenges and Opportunities in the Development of Conjugated Block Copolymers for Photovoltaics. Macromolecules 2015, 48, 7385.

(24) Sommer, M.; Huettner, S.; Thelakkat, M. Donor-acceptor block copolymers for photovoltaic applications. J. Mater. Chem. 2010, 20, 10788 .

(25) Madhu, M.; Ramakrishnan, R.; Vijay, V.; Hariharan, M. Free Charge Carriers in Homo-Sorted $\pi$-Stacks of Donor-Acceptor Conjugates. Chem. Rev. 2021, 121, 8234.

(26) Bu, L.; Guo, X.; Yu, B.; Qu, Y.; Xie, Z.; Yan, D.; Geng, Y.; Wang, F. Monodisperse Co-oligomer Approach toward Nanostructured Films with Alternating Donor-Acceptor Lamellae. J. Am. Chem. Soc. 2009, $131,13242$.

(27) Qu, J.; Gao, B.; Tian, H.; Zhang, X.; Wang, Y.; Xie, Z.; Wang, H.; Geng, Y.; Wang, F. Donor-spacer-acceptor monodisperse conjugated co-oligomers for efficient single-molecule photovoltaic cells based on non-fullerene acceptors. J. Mater. Chem. A 2014, 2, 3632. 
(28) Schwartz, P. O.; Biniek, L.; Zaborova, E.; Heinrich, B.; Brinkmann, M.; Leclerc, N.; Méry, S. Perylenediimide-Based DonorAcceptor Dyads and Triads: Impact of Molecular Architecture on SelfAssembling Properties. J. Am. Chem. Soc. 2014, 136, 5981.

(29) Biniek, L.; Schwartz, P. O.; Zaborova, E.; Heinrich, B.; Leclerc, N.; Méry, S.; Brinkmann, M. Zipper-like molecular packing of donoracceptor conjugated co-oligomers based on perylenediimide. J. Mater. Chem. C 2015, 3, 3342.

(30) D’Avino, G.; Muccioli, L.; Castet, F.; Poelking, C.; Andrienko, D.; Soos, Z. G.; Cornil, J.; Beljonne, D. Electrostatic phenomena in organic semiconductors: fundamentals and implications for photovoltaics. J. Phys.: Condens. Matter 2016, 28, 433002.

(31) Liu, L.; Eisenbrandt, P.; Roland, T.; Polkehn, M.; Schwartz, P. O.; Bruchlos, K.; Omiecienski, B.; Ludwigs, S.; Leclerc, N.; Zaborova, E.; et al. Controlling charge separation and recombination by chemical design in donor-acceptor dyads. Phys. Chem. Chem. Phys. 2016, 18, 18536.

(32) Méry, S.; Haacke, S. Private Communication, 2021.

(33) Farkas, L. Keimbildungsgeschwindigkeit in übersättigten Dämpfen. Z. Phys. Chem. 1927, 125U, 236.

(34) Kramers, H. A. Brownian motion in a field of force and the diffusion model of chemical reactions. Physica 1940, 7, 284.

(35) Hänggi, P.; Talkner, P.; Borkovec, M. Reaction-rate theory: fifty years after Kramers. Rev. Mod. Phys. 1990, 62, 251.

(36) Takahashi, Y.; Umezawa, H. Thermo field dynamics. Collect. Phenom. 1975, 2, 55.

(37) Takahashi, Y.; Umezawa, H. Thermo field dynamics. Int. J. Mod. Phys. B 1996, 10, 1755.

(38) Barnett, S. M.; Knight, P. L. Thermofield analysis of squeezing and statistical mixtures in quantum optics. J. Opt. Soc. Am. B 1985, 2, 467.

(39) Borrelli, R.; Gelin, M. F. Quantum electron-vibrational dynamics at finite temperature: Thermo field dynamics approach. J. Chem. Phys. 2016, 145, 224101.

(40) Borrelli, R.; Gelin, M. F. Simulation of Quantum Dynamics of Excitonic Systems at Finite Temperature: an efficient method based on Thermo Field Dynamics. Sci. Rep. 2017, 7, 9127.

(41) Borrelli, R.; Gelin, M. Finite Temperature Quantum Dynamics of Complex Systems: Integrating Thermo-Field Theories and TensorTrain Methods. Wiley Interdiscip. Rev.: Comput. Mol. Sci. 2021, 11, No. e1539.

(42) Wang, H.; Thoss, M. Multilayer formulation of the multiconfiguration time-dependent Hartree theory. J. Chem. Phys. 2003, 119, 1289.

(43) Manthe, U. A multilayer multiconfigurational time-dependent Hartree approach for quantum dynamics on general potential energy surfaces. J. Chem. Phys. 2008, 128, 164116.

(44) Vendrell, O.; Meyer, H. Multilayer multiconfiguration timedependent Hartree method: Implementation and applications to a Henon-Heiles Hamiltonian and to pyrazine. J. Chem. Phys. 2011, 134, 044135 .

(45) Wang, H. Multilayer Multiconfiguration Time-Dependent Hartree Theory. J. Phys. Chem. A 2015, 119, 7951.

(46) Beck, M. H.; Jäckle, A.; Worth, G. A.; Meyer, H.-D. The multiconfiguration time-dependent Hartree (MCTDH) method: a highly efficient algorithm for propagating wavepackets. Phys. Rep. 2000, $324,1$.

(47) Köuppel, H.; Domcke, W.; Cederbaum, L. S. Multimode Molecular Dynamics Beyond the Born-Oppenheimer Approximation. Adv. Chem. Phys. 2007, 57, 59.

(48) Rolland, D.; Brauer, J. C.; Hartmann, L.; Biniek, L.; Brinkmann, M.; Banerji, N.; Frauenrath, H. Charge separation in an acceptordonor-acceptor triad material with a lamellar structure. J. Mater. Chem. C 2017, 5, 1383.

(49) Logsdon, J. L.; Hartnett, P. E.; Nelson, J. N.; Harris, M. A.; Marks, T. J.; Wasielewski, M. R. Charge Separation Mechanisms in Ordered Films of Self-Assembled Donor-Acceptor Dyad Ribbons. ACS Appl. Mater. Interfaces 2017, 9, 33493.
(50) Roland, T.; Léonard, J.; Hernandez Ramirez, G.; Méry, S.; Yurchenko, O.; Ludwigs, S.; Haacke, S. Sub-100 fs charge transfer in a novel donor-acceptor-donor triad organized in a smectic film. Phys. Chem. Chem. Phys. 2012, 14, 273.

(51) Polkehn, M.; Tamura, H.; Eisenbrandt, P.; Haacke, S.; Méry, S.; Burghardt, I. Molecular Packing Determines Charge Separation in a Liquid Crystalline Bisthiophene-Perylene Diimide Donor-Acceptor Material. J. Phys. Chem. Lett. 2016, 7, 1327.

(52) Nübling, F.; Hopper, T. R.; Kuei, B.; Komber, H.; Untilova, V.; Schmidt, S. B.; Brinkmann, M.; Gomez, E. D.; Bakulin, A. A.; Sommer, M. Block Junction-Functionalized All-Conjugated Donor-Acceptor Block Copolymers. ACS Appl. Mater. Interfaces 2019, 11, 1143.

(53) Blancafort, L.; Voityuk, A. Exciton delocalization, charge transfer, and electronic coupling for singlet excitation energy transfer between stacked nucleobases in DNA: An MS-CASPT2 study. J. Chem. Phys. 2014, 140, 095102.

(54) Geng, Y.; Wang, J.; Wu, S.; Li, H.; Yu, F.; Yang, G.; Gao, H.; Su, Z. Theoretical discussions on electron transport properties of perylene bisimide derivatives with different molecular packings and intermolecular interactions. J. Mater. Chem. 2011, 21, 134.

(55) Seidner, L.; Stock, G.; Sobolewski, A. L.; Domcke, W. Ab initio characterization of the $\mathrm{S} 1-\mathrm{S} 2$ conical intersection in pyrazine and calculation of spectra. J. Chem. Phys. 1992, 96, 5298.

(56) Cederbaum, L. S.; Gindensperger, E.; Burghardt, I. Short-Time Dynamics Through Conical Intersections in Macrosystems. Phys. Rev. Lett. 2005, 94, 113003.

(57) Tamura, H.; Ramon, J.; Bittner, E. R.; Burghardt, I. PhononDriven Ultrafast Exciton Dissociation at Donor-Acceptor Polymer Heterojunctions. Phys. Rev. Lett. 2008, 100, 107402.

(58) Burghardt, I.; Nest, M.; Worth, G. A. Multiconfigurational system-bath dynamics using Gaussian wave packets: Energy relaxation and decoherence induced by a finite-dimensional bath. J. Chem. Phys. 2003, 119, 5364.

(59) Tamura, H.; Martinazzo, R.; Ruckenbauer, M.; Burghardt, I. Quantum dynamics of ultrafast charge transfer at an oligothiophenefullerene heterojunction. J. Chem. Phys. 2012, 137, 22 A540.

(60) Bonfanti, M.; Jackson, B.; Hughes, K. H.; Burghardt, I.; Martinazzo, R. Quantum dynamics of hydrogen atoms on graphene. I. System-bath modeling. J. Chem. Phys. 2015, 143, 124703.

(61) van Kampen, N. G. Stochastic Processes in Physics and Chemistry; North-Holland: Amsterdam, 1992.

(62) Borrelli, R. Theoretical Study of Charge-Transfer Processes at Finite Temperature Using a Novel Thermal Schroedinger Equation. Chem. Phys. 2018, 515, 236.

(63) Chen, L.; Borrelli, R.; Shalashilin, D. V.; Zhao, Y.; Gelin, M. Simulation of Time- and Frequency-Resolved Four-Wave-Mixing Signals at Finite Temperatures: A Thermo-Field Dynamics Approach. J. Chem. Theory Comput. 2021, 17, 4359.

(64) Borrelli, R.; Gelin, M. Simulation of Nonlinear Femtosecond Signals at Finite Temperature via a Thermo Field Dynamics-Tensor Train Method: General Theory and Application to Time- and Frequency-Resolved Fluorescence of the Fenna-Matthews-Olson Complex. J. Chem. Theory Comput. 2021, 17, 4316.

(65) Bogoljubov, N. N. On a new method in the theory of superconductivity. Nuovo Cimento 1958, 7, 794.

(66) Meyer, H.-D.; Manthe, U.; Cederbaum, L. S. The multiconfigurational time-dependent Hartree approach. Chem. Phys. Lett. 1990, 165, 73.

(67) Manthe, U.; Meyer, H.-D.; Cederbaum, L. S. Wave-packet dynamics within the multiconfiguration Hartree framework: General aspects and application to NOCl. J. Chem. Phys. 1992, 97, 3199.

(68) Worth, G. A.; Beck, M. H.; Jäckle, A.; Meyer, H.-D. MCTDH Package, version 8.5.5; Universität Heidelberg, 2016. http://www. mctdh.uni-hd.de.

(69) Chen, L.; Shi, Q. Quantum rate dynamics for proton transfer reactions in condensed phase: The exact hierarchical equations of motion approach. J. Chem. Phys. 2009, 130, 134505.

(70) Lehmann, J.; Reimann, P.; Hänggi, P. Surmounting Oscillating Barriers. Phys. Rev. Lett. 2000, 84, 1639. 
(71) Huix-Rotllant, M.; Tamura, H.; Burghardt, I. Concurrent effects of delocalization and internal conversion tune charge separation at donor-acceptor polymer heterojunctions. J. Phys. Chem. Lett. 2015, 6, 1702 .

(72) Landi, A.; Borrelli, R.; Capobianco, A.; Velardo, A.; Peluso, A. Hole Hopping Rates in Organic Semiconductors: A Second-Order Cumulant Approach. J. Chem. Theory Comput. 2018, 14, 1594.

(73) Landi, A.; Borrelli, R.; Capobianco, A.; Velardo, A.; Peluso, A. Second-Order Cumulant Approach for the Evaluation of Anisotropic Hole Mobility in Organic Semiconductors. J. Phys. Chem. C 2018, 122, 25849.

(74) Schmidt, R.; Oh, J. H.; Sun, Y.; Deppisch, M.; Krause, A.; Radacki, K.; Braunschweig, H.; Könemann, M.; Erk, P.; Bao, Z.; Würthner, F. High-performance air-stable n-channel organic thin film transistors based on halogenated perylene bisimide semiconductors. J. Am. Chem. Soc. 2009, 131, 6215.

(75) Bera, S.; Gheeraert, N.; Fratini, S.; Ciuchi, S.; Florens, S. Impact of quantized vibrations on the efficiency of interfacial charge separation in photovoltaic devices. Phys. Rev. B: Condens. Matter Mater. Phys. 2015, 91, No. 041107.

(76) Richler, K.; Mayou, D. Influence of static disorder and polaronic band formation on the interfacial electron transfer in organic photovoltaic devices. Phys. Rev. B: Condens. Matter Mater. Phys. 2019, 99, 195151.

(77) Barth, S.; Bässler, H.; Scherf, U.; Müllen, K. Photoconduction in thin films of a ladder-type poly-para-phenylene. Chem. Phys. Lett. 1998, $288,147$.

(78) Christ, N.; Kettlitz, S. W.; Valouch, S.; Mescher, J.; Nintz, M.; Lemmer, U. Intensity dependent but temperature independent charge carrier generation in organic photodiodes and solar cells. Org. Electron. 2013, 14, 973.

(79) Son, M.; Park, K. H.; Shao, C.; Würthner, F.; Kim, D. Spectroscopic Demonstration of Exciton Dynamics and Excimer Formation in a Sterically Controlled Perylene Bisimide Dimer Aggregate. J. Phys. Chem. Lett. 2014, 5, 3601.

(80) Singh, R.; Kim, M.; Lee, J.-J.; Ye, T.; Keivanidis, P. E.; Cho, K. Excimer formation effects and trap-assisted charge recombination loss channels in organic solar cells of perylene diimide dimer acceptors. $J$. Mater. Chem. C 2020, 8, 1686-1696. 